\title{
Robust Adaptive Relative Position and Attitude Control for Spacecraft Autonomous Proximity
}

\begin{abstract}
This paper provides new results of the dynamical modeling and controller designing for autonomous close proximity phase during rendezvous and docking in the presence of kinematic couplings and model uncertainties. A globally defined relative motion mechanical model for close proximity operations is introduced firstly. Then, in spite of the kinematic couplings and thrust misalignment between relative rotation and relative translation, robust adaptive relative position and relative attitude controllers are designed successively. Finally, stability of the overall system is proved that the relative position and relative attitude are uniformly ultimately bounded, and the size of the ultimate bound can be regulated small enough by control system parameters. Performance of the controlled overall system is demonstrated via a representative numerical example.

Keywords: Spacecraft control, close proximity operations, kinematic couplings, thrust misalignment, adaptive control, robust control.
\end{abstract}

\section{Introduction}

Spacecraft proximity control is an essential and important problem and has been widely studied in many space missions, such as spacecraft rendezvous and docking, on-orbit servicing, fuel supplying, formation flying, and space station 5 construction. Generally, two rigid spacecraft, namely a chaser and a target, are involved in close proximity missions. The relative position tracking and attitude synchronization between two spacecraft are required simultaneously in close proximity scenario. Compared with the rigid body position control in three-dimension space, rigid body attitude control is a more difficult problem

Preprint submitted to ISA Transactions

December 17, 2015

(C) 2016. This manuscript version is made available under the Elsevier user license http://www.elsevier.com/open-access/userlicense/1.0/ 
that plays a central role in many mechanical system applications and has therefore received considerable attention over the years. An adaptive fault-tolerant attitude controller was proposed in [1], and finite-time attitude controllers were redesigned in [2] and [3] based on pulse modulation synthesis and the adaptive fuzzy control method, respectively. A time-varying terminal sliding mode technique was used to design the finite-time attitude tracking controller in [4]. A fixed-time attitude tracking controller was developed in [5] based on planning the desired quaternion attitude trajectory. Except for the attitude control for single spacecraft, there are many attitude synchronization controllers for spacecraft formation, such as neural network-based terminal sliding mode distributed controller [6], adaptive nonsingular fast terminal sliding mode-based obstacle avoidance controller [7], extended Kalman filter-based fault detection and isolation controller [8], and distributed controller without angular velocities measurement [9]. The classical method for the stabilization and tracking of rigid bodies in attitude relies on a local parametrization of the rotation matrix[10], such as the Euler angles, quaternion or Rodrigues parameters. Compared with position dynamics, the distinct features of the attitude dynamics is that its state space is not linear[11]. This yields important and unique properties that can not be observed from dynamic systems evolving on a linear space. Even though the state space for attitude control problem is nonlinear, this problem 30 has been analyzed by using coordinate representations resulting in different attitude kinematics[12]. Recently, a coordinate-free approach has been proposed to deal with the attitude dynamics directly based on the rotation matrix[13]. One of the main advantages of this approach is that it allows a global analysis of the designed feedback control law. However, it has been proved that global asymptotic stability is not achievable through continuous feedback, and the notion of almost global asymptotic stability is the best achievement in continuous attitude control[14].

Many models and controllers for spacecraft relative motion have been developed from the control community in theory and practice. To the best of our knowledge, the existing literatures on relative attitude control in formation 
or proximity operations are all based on the coordinate-dependent approach. Quaternion is employed to establish the relative attitude dynamics in [15]-[20]. Modified Rodrigues parameters are used to model the attitude dynamics in [21]-[24]. Moreover, dual quaternion was proposed for relative attitude and rel45 ative position motions in [25]-[27]. Recently, based on the quaternion attitude dynamics, an inverse optimal sliding mode controller was designed for spacecraft relative pose motion in [28]. Except for singularities of quaternion and modified Rodrigues parameters, they also have ambiguities in representing an attitude. For instance, in a quaternion-based attitude control system, convergence to a single attitude implies convergence to either of the two disconnected and antipodal points[29]. Otherwise, it may also exhibit unwinding behavior, where the controller rotates a rigid body through unnecessarily large angles[14]. An adaptive sliding mode relative pose controller was designed directly on the Lie group in [30], but the relative pose model is assumed well known without uncertainties. Above schemes are available to establish the relative motion model and realize the spacecraft close proximity operations, but most of them have following two drawbacks:(i) the mechanical model of the relative motion between two spacecraft is not globally defined; (ii) the inherently kinematic couplings between relative position and relative attitude motions, mass and inertia parametric uncertainties of two spacecraft, unknown thrust misalignment, and bounded external disturbances in the mechanical model are not considered simultaneously in the controller design. In order to dealing with these two challenges, this study focus on the new development of the modeling and control for spacecraft autonomous proximity operations. The unique features of this study lies in following aspects.

- Compared with the coordinate-dependent relative attitude controller in [15]-[24], relative attitude controller in this paper is developed directly based on the rotation matrix to avoid the singularities associated with coordinate-dependent representations, a globally defined nonlinear relative motion model is presented for close proximity operations by using the 
coordinate-free approach, and compared with the model in [31]-[34], the gravity of two spacecraft has been considered in relative position dynamics.

- With treating the kinematic couplings and external disturbances as bounded lumped disturbances for relative rotational and relative translational systems, the relative position and relative attitude controllers are designed one after another based on robust adaptive control method, where the element-wise adaptive laws are used to estimate unknown mass and inertia of the chaser, the norm-wise adaptive laws are used to estimate thrust misalignment vector and the upper bounds of lumped disturbances. Specially, the amount of online estimate parameters in the proposed adaptive controllers is only 12 , thus the computational burden of the controller is largely decreased for the spacecraft proximity operations compared with the larger amount 392 in [35] and 324 in [36].

- A rigorous Lyapunov analysis, that explicitly considers the kinematic couplings and model uncertainties in relative dynamics, is presented to establish stability properties, then uniformly ultimately boundedness of relative position and relative attitude is derived, and the ultimate bounds can be regulated sufficiently small with appropriate controller parameters. Moreover, the discontinuous signum function in [31][32] is replaced by hyperbolic tangent function to avoid the high-frequency chattering phenomenon in controllers.

The rest of this paper is organized as follows. Section 2 gives some notations and properties used in this paper. Section 3 derives a global mechanical model for proximity operations and summarizes an objective of the controller design. Section 4 proposes the controller design and stability analysis of two closed-loop subsystems. Section 5 verifies the application of the controller to a scenario of autonomous proximity operations. Section 6 concludes the work. 


\section{Preliminaries}

Throughout this paper, the skew symmetric matrix $S(\boldsymbol{a}) \in \mathbb{R}^{3 \times 3}$ derived from a vector $\boldsymbol{a}=\left[a_{1}, a_{2}, a_{3}\right]^{\mathrm{T}} \in \mathbb{R}^{3}$ is defined as

$$
S(\boldsymbol{a})=\left[\begin{array}{ccc}
0 & -a_{3} & a_{2} \\
a_{3} & 0 & -a_{1} \\
-a_{2} & a_{1} & 0
\end{array}\right] .
$$

Actually, $S(\cdot)$ is a map $\mathbb{R}^{3} \rightarrow s o(3)$, this identifies the Lie algebra $s o(3)$ with $\mathbb{R}^{3}$ using the vector cross product in $\mathbb{R}^{3}$. The inverse of the map $S(\cdot)$ is referred to as the vee map, that is $\mathrm{V}: s o(3) \rightarrow \mathbb{R}^{3}$. Some properties of the map $S(\cdot)$ are listed as follows[11]:

$$
\begin{gathered}
S(\boldsymbol{a}) \boldsymbol{b}=\boldsymbol{a} \times \boldsymbol{b}=-\boldsymbol{b} \times \boldsymbol{a}=-S(\boldsymbol{b}) \boldsymbol{a}, \\
\|S(\boldsymbol{a})\|=\|\boldsymbol{a}\|, \boldsymbol{a}^{\mathrm{T}} S(\boldsymbol{a})=\mathbf{0}, \boldsymbol{a}^{\mathrm{T}} S(\boldsymbol{b}) \boldsymbol{a}=0, \\
S(\boldsymbol{a})=-S^{\mathrm{T}}(\boldsymbol{a}),-\frac{1}{2} \operatorname{tr}(S(\boldsymbol{a}) S(\boldsymbol{b}))=\boldsymbol{a}^{\mathrm{T}} \boldsymbol{b}, \\
\operatorname{tr}(S(\boldsymbol{a}) A)=\operatorname{tr}(A S(\boldsymbol{a}))=-\boldsymbol{a}^{\mathrm{T}}\left(A-A^{\mathrm{T}}\right)^{\vee}, \\
S(\boldsymbol{a}) A+A^{\mathrm{T}} S(\boldsymbol{a})=S\left(\left[\operatorname{tr}(A) I_{3}-A\right] \boldsymbol{a}\right) ; R S(\boldsymbol{a}) R^{\mathrm{T}}=S(R \boldsymbol{a}),
\end{gathered}
$$

for any $\boldsymbol{a}, \boldsymbol{b} \in \mathbb{R}^{3}, A \in \mathbb{R}^{3 \times 3}$, and $R \in \mathrm{SO}(3)$. Moreover, $\operatorname{tr}(A)$ denotes the matrix trace; $\|\boldsymbol{a}\|$ and $\|\boldsymbol{a}\|_{1}$ denote vector 2-norm and vector 1-norm; $\|A\|$ and $\|A\|_{F}$ are induced matrix 2-norm and Frobenius-norm; $\mathrm{SO}(3) \triangleq\left\{R \in \mathbb{R}^{3 \times 3}\right.$ : $R^{\mathrm{T}} R=I_{3} ; \operatorname{det}(R)=1$ \} denotes the special orthogonal group; $I_{n}$ and $O_{n}$ are $n \times n$ unit and zero matrices, respectively.

Define $\tanh (\boldsymbol{a})=\left[\tanh \left(a_{1}\right), \tanh \left(a_{2}\right), \tanh \left(a_{3}\right)\right]^{\mathrm{T}}$, where the hyperbolic tangent function $\tanh \left(a_{i}\right)=\frac{\mathrm{e}^{a_{i}}-\mathrm{e}^{-a_{i}}}{\mathrm{e}^{a_{i}}+\mathrm{e}^{-a_{i}}}, i=1,2,3$, and it satisfies $\left|\tanh \left(a_{i}\right)\right|<1$, $\tanh \left(a_{i}\right)=0$ if and only if $a_{i}=0$. Moreover, the following inequality holds for any constant $c>0$ and $a_{i} \in \mathbb{R}[37]$ :

$$
0 \leq\left|a_{i}\right|-a_{i} \tanh \left(c a_{i}\right) \leq \frac{\epsilon}{c}
$$

where $\epsilon$ is a constant that satisfies $\epsilon=\mathrm{e}^{-(\epsilon+1)}$, i.e., $\epsilon=0.2785$. 


\section{Problem Statement}

\subsection{Chaser and Target Dynamics}

The control problem that a chaser subject to uncertain inertia and unknown disturbance tracks a tumbling non-cooperative space target is considered in this study. Figure 1 depicts the related frames and vectors, where $\mathcal{F}_{i} \triangleq\left\{\mathbf{O} x_{i} y_{i} z_{i}\right\}$ represents an Earth-centered inertia frame, $\mathcal{F}_{c} \triangleq\{\mathbf{C} x y z\}$ and $\mathcal{F}_{t} \triangleq\left\{\mathbf{T} x_{t} y_{t} z_{t}\right\}$ are the chaser and target body-fixed frames; $\mathbf{O}, \mathbf{C}$, and $\mathbf{T}$ are the centers of mass of the Earth, chaser, and target, respectively; $\mathbf{P}$ is the chaser desired approaching position along the direction of target docking port; $\left\{\boldsymbol{r}, \boldsymbol{r}_{e}\right\}$ and $\left\{\boldsymbol{r}_{t}, \boldsymbol{p}_{t}, \boldsymbol{r}_{p t}\right\}$ are the related position vectors represented in frame $\mathcal{F}_{c}$ and frame $\mathcal{F}_{t}$, respectively. The aim of this study is to design controller such that origin $\mathbf{C}$ tracks point $\mathbf{P}$ and frame $\mathcal{F}_{c}$ tracks frame $\mathcal{F}_{t}$.

The position motion of center of mass $\mathbf{C}$ and the attitude motion of frame $\mathcal{F}_{c}$ with respect to frame $\mathcal{F}_{i}$ can be described by following chaser kinematics and dynamics equations expressed in frame $\mathcal{F}_{c}[38]$ :

$$
\dot{R}=R S(\boldsymbol{\omega})
$$

120

$$
\begin{gathered}
\dot{\boldsymbol{r}}=\boldsymbol{v}-S(\boldsymbol{\omega}) \boldsymbol{r}, \\
J \dot{\boldsymbol{\omega}}+S(\boldsymbol{\omega}) J \boldsymbol{\omega}=\boldsymbol{\tau}+S(\boldsymbol{\rho}) \boldsymbol{f}+\boldsymbol{d}_{\tau}, \\
m \dot{\boldsymbol{v}}+m S(\boldsymbol{\omega}) \boldsymbol{v}+\mu m \boldsymbol{r}=\boldsymbol{f}+\boldsymbol{d}_{f},
\end{gathered}
$$

where $\boldsymbol{r} \in \mathbb{R}^{3}$ is chaser's position and $R \in \mathrm{SO}(3)$ denotes chaser's attitude; $\boldsymbol{v}$ and $\boldsymbol{\omega} \in \mathbb{R}^{3}$ are velocity and angular velocity of the chaser, respectively; ${ }_{125} \mu=\mu_{g} /\|\boldsymbol{r}\|^{3}, \mu_{g}$ is the gravitational constant of the Earth; $\boldsymbol{\tau}$ and $\boldsymbol{f} \in \mathbb{R}^{3}$ are control force and torque, respectively; $\boldsymbol{\rho} \in \mathbb{R}^{3}$ is the thrust misalignment vector from the center of mass $\mathbf{C}$ to the applied point of force $\boldsymbol{f} ; \boldsymbol{d}_{f}$ and $\boldsymbol{d}_{\tau} \in \mathbb{R}^{3}$ are disturbance force and torque, respectively; $m \in \mathbb{R}$ and $J \in \mathbb{R}^{3 \times 3}$ are the chaser mass and inertia matrix, respectively. 
Similarly, consider that target moves in the circular orbit. With only considering the gravity and ignoring the other external forces and torques, kinematics and dynamics of the tumbling target can be modeled in frame $\mathcal{F}_{t}$ as follows [39]:

$$
\begin{gathered}
\dot{R}_{t}=R_{t} S\left(\boldsymbol{\omega}_{t}\right), \\
\dot{\boldsymbol{r}}_{t}=\boldsymbol{v}_{t}-S\left(\boldsymbol{\omega}_{t}\right) \boldsymbol{r}_{t}, \\
J_{t} \dot{\boldsymbol{\omega}}_{t}+S\left(\boldsymbol{\omega}_{t}\right) J_{t} \boldsymbol{\omega}_{t}=\mathbf{0},
\end{gathered}
$$

135

$$
m_{t} \dot{\boldsymbol{v}}_{t}+m_{t} S\left(\boldsymbol{\omega}_{t}\right) \boldsymbol{v}_{t}+\mu_{t} m_{t} \boldsymbol{r}_{t}=\mathbf{0}
$$

where $\boldsymbol{r}_{t} \in \mathbb{R}^{3}$ is target's position and $R_{t} \in \mathrm{SO}(3)$ denotes target's attitude; $\boldsymbol{v}_{t}$ and $\boldsymbol{\omega}_{t} \in \mathbb{R}^{3}$ are velocity and angular velocity of the target, respectively; $\mu_{t}=\mu_{g} / r_{o}^{3}, r_{o}$ is the radius of the circular orbit; $m_{t} \in \mathbb{R}$ and $J_{t} \in \mathbb{R}^{3 \times 3}$ are target's mass and positive definite symmetric inertia matrix, respectively.

Remark 1 Since the external forces and torques are ignored except for the gravity in target dynamics, then the radius of the target's circular orbit $r_{o}$ is a positive constant, and scalar $\mu_{t}$ is also a positive constant. By defining kinetic energy function $E_{n}(t)=\frac{1}{2}\left(\mu_{t} m_{t} \boldsymbol{r}_{t}^{\mathrm{T}} \boldsymbol{r}_{t}+m_{t} \boldsymbol{v}_{t}^{\mathrm{T}} \boldsymbol{v}_{t}+\boldsymbol{\omega}_{t}^{\mathrm{T}} J_{t} \boldsymbol{\omega}_{t}\right) \geq 0$ and calculating its time derivative $\dot{E}_{n}(t)=-\mu_{t} m_{t} \boldsymbol{r}_{t}^{\mathrm{T}} S\left(\boldsymbol{\omega}_{t}\right) \boldsymbol{r}_{t}-m_{t} \boldsymbol{v}_{t}^{\mathrm{T}} S\left(\boldsymbol{\omega}_{t}\right) \boldsymbol{v}_{t}-\boldsymbol{\omega}_{t}^{\mathrm{T}} S\left(\boldsymbol{\omega}_{t}\right) J_{t} \boldsymbol{\omega}_{t} \equiv 0$, we know $E_{n}(t) \equiv E_{n}(0)=\frac{1}{2}\left[\mu_{t} m_{t} \boldsymbol{r}_{t}^{\mathrm{T}}(0) \boldsymbol{r}_{t}(0)+m_{t} \boldsymbol{v}_{t}^{\mathrm{T}}(0) \boldsymbol{v}_{t}(0)+\boldsymbol{\omega}_{t}^{\mathrm{T}}(0) J_{t} \boldsymbol{\omega}_{t}(0)\right]<$ $\infty$. Thus, the tumbling target's position $\boldsymbol{r}_{t}$, velocity $\boldsymbol{v}_{t}$ and angular velocity $\boldsymbol{\omega}_{t}$ are always bounded.

\subsection{Relative Position Dynamics}

In light of Figure 1, the position and velocity of point $\mathbf{P}$ denoted in frame $\mathcal{F}_{t}$ are

$$
\left\{\begin{array}{l}
\boldsymbol{r}_{p_{t}}=\boldsymbol{r}_{t}+\boldsymbol{p}_{t} \\
\boldsymbol{v}_{p_{t}}=\boldsymbol{v}_{t}+S\left(\boldsymbol{\omega}_{t}\right) \boldsymbol{p}_{t},
\end{array}\right.
$$


where the $\boldsymbol{p}_{t} \in \mathbb{R}^{3}$ is a constant vector denoted in frame $\mathcal{F}_{t}$. The relative position, relative velocity and relative angular velocity are also denoted in frame $\mathcal{F}_{c}$ by

$$
\left\{\begin{array}{l}
\boldsymbol{r}_{e}=\boldsymbol{r}-R_{e}^{\mathrm{T}} \boldsymbol{r}_{p_{t}}, \\
\boldsymbol{v}_{e}=\boldsymbol{v}-R_{e}^{\mathrm{T}} \boldsymbol{v}_{p_{t}} \\
\boldsymbol{\omega}_{e}=\boldsymbol{\omega}-R_{e}^{\mathrm{T}} \boldsymbol{\omega}_{t}
\end{array}\right.
$$

where $R_{e}=R_{t}^{\mathrm{T}} R \in \mathrm{SO}(3)$ is the rotation matrix from $\mathcal{F}_{c}$ to $\mathcal{F}_{t}$.

Substituting (10) into (2) and (4), and using $\dot{\boldsymbol{r}}_{p_{t}}=\boldsymbol{v}_{p_{t}}-S\left(\boldsymbol{\omega}_{t}\right) \boldsymbol{r}_{p_{t}}$ yield the relative position motion model as

$$
\begin{gathered}
\dot{\boldsymbol{r}}_{e}=\boldsymbol{v}_{e}-S(\boldsymbol{\omega}) \boldsymbol{r}_{e}, \\
m \dot{\boldsymbol{v}}_{e}=-m\left[S(\boldsymbol{\omega}) \boldsymbol{v}+\mu \boldsymbol{r}+R_{e}^{\mathrm{T}} \dot{\boldsymbol{v}}_{p_{t}}-S\left(\boldsymbol{\omega}_{e}\right)\left(\boldsymbol{v}-\boldsymbol{v}_{e}\right)\right]+\boldsymbol{f}+\boldsymbol{d}_{f},
\end{gathered}
$$

where $R_{e}^{\mathrm{T}} \dot{\boldsymbol{v}}_{p_{t}}$ can be derived from (8), (9), (10) and $R_{e}^{\mathrm{T}} S(\boldsymbol{a})=S\left(R_{e}^{\mathrm{T}} \boldsymbol{a}\right) R_{e}^{\mathrm{T}}$ for any $\boldsymbol{a} \in \mathbb{R}^{3}$ as

$$
\begin{aligned}
R_{e}^{\mathrm{T}} \dot{\boldsymbol{v}}_{p_{t}}= & R_{e}^{\mathrm{T}}\left[\dot{\boldsymbol{v}}_{t}+S\left(\dot{\boldsymbol{\omega}}_{t}\right) \boldsymbol{p}_{t}\right]=R_{e}^{\mathrm{T}}\left[-S\left(\boldsymbol{\omega}_{t}\right) \boldsymbol{v}_{t}-\mu_{t} \boldsymbol{r}_{t}-S\left(\boldsymbol{p}_{t}\right) \dot{\boldsymbol{\omega}}_{t}\right] \\
= & -S\left(R_{e}^{\mathrm{T}} \boldsymbol{\omega}_{t}\right)\left[R_{e}^{\mathrm{T}} \boldsymbol{v}_{p_{t}}-R_{e}^{\mathrm{T}} S\left(\boldsymbol{\omega}_{t}\right) \boldsymbol{p}_{t}\right]-\mu_{t} R_{e}^{\mathrm{T}} \boldsymbol{r}_{t}-R_{e}^{\mathrm{T}} S\left(\boldsymbol{p}_{t}\right) \dot{\boldsymbol{\omega}}_{t} \\
= & -S\left(\boldsymbol{\omega}-\boldsymbol{\omega}_{e}\right)\left[\boldsymbol{v}-\boldsymbol{v}_{e}-S\left(\boldsymbol{\omega}-\boldsymbol{\omega}_{e}\right) R_{e}^{\mathrm{T}} \boldsymbol{p}_{t}\right] \\
& +\mu_{t} R_{e}^{\mathrm{T}} \boldsymbol{p}_{t}-\mu_{t}\left(\boldsymbol{r}-\boldsymbol{r}_{e}\right)-R_{e}^{\mathrm{T}} S\left(\boldsymbol{p}_{t}\right) \dot{\boldsymbol{\omega}}_{t} .
\end{aligned}
$$

Thus relative position dynamics (12) can be rewritten as

$$
m \dot{\boldsymbol{v}}_{e}=-m \boldsymbol{g}-\boldsymbol{n}_{P}+\boldsymbol{f}+\boldsymbol{d}_{f},
$$

where $\boldsymbol{g}=S(\boldsymbol{\omega}) \boldsymbol{v}_{e}+S^{2}\left(\boldsymbol{\omega}-\boldsymbol{\omega}_{e}\right) R_{e}^{\mathrm{T}} \boldsymbol{p}_{t}+\mu \boldsymbol{r}+\mu_{t} R_{e}^{\mathrm{T}} \boldsymbol{p}_{t}-\mu_{t}\left(\boldsymbol{r}-\boldsymbol{r}_{\boldsymbol{e}}\right)$ and $\boldsymbol{n}_{P}=$ ${ }_{155} \quad m R_{e}^{\mathrm{T}} S\left(\boldsymbol{p}_{t}\right) J_{t}^{-1} S\left(\boldsymbol{\omega}_{t}\right) J_{t} \boldsymbol{\omega}_{t}$.

\subsection{Relative Attitude Dynamics}

Define a relative attitude trace function $\Psi \in \mathbb{R}$ and a relative attitude vector $e_{R} \in \mathbb{R}^{3}$ as

$$
\left\{\begin{array}{l}
\Psi\left(R_{e}\right)=\frac{1}{2} \operatorname{tr}\left(K-K R_{e}\right) \\
e_{R}\left(R_{e}\right)=\frac{1}{2}\left(K R_{e}-R_{e}^{\mathrm{T}} K\right)^{\mathrm{V}}
\end{array}\right.
$$


where $K=\operatorname{diag}\left\{k_{1}, k_{2}, k_{3}\right\}$ is a diagonal matrix with distinct and positive constants $k_{i}(i=1,2,3)$. It has been proved in [40] that $\Psi\left(R_{e}\right)$ is locally positive definite and satisfies

$$
b_{1}\left\|\boldsymbol{e}_{R}\left(R_{e}\right)\right\|^{2} \leq\left\|\Psi\left(R_{e}\right)\right\| \leq b_{2}\left\|\boldsymbol{e}_{R}\left(R_{e}\right)\right\|^{2}
$$

with the constants $b_{1}=\frac{h_{1}}{h_{2}+h_{3}}$ and $b_{2}=\frac{h_{1} h_{4}}{h_{5}\left(h_{1}-b_{0}\right)}$, where $b_{0}$ is a positive constant that strictly satisfies $\Psi\left(R_{e}\right)<b_{0}<h_{1}$, and $h_{i}(i=1, \cdots, 5)$ are defined by

$$
\begin{aligned}
& h_{1}=\min \left\{k_{1}+k_{2}, k_{2}+k_{3}, k_{3}+k_{1}\right\}, \\
& h_{2}=\max \left\{\left(k_{1}-k_{2}\right)^{2},\left(k_{2}-k_{3}\right)^{2},\left(k_{3}-k_{1}\right)^{2}\right\}, \\
& h_{3}=\max \left\{\left(k_{1}+k_{2}\right)^{2},\left(k_{2}+k_{3}\right)^{2},\left(k_{3}+k_{1}\right)^{2}\right\}, \\
& h_{4}=\max \left\{k_{1}+k_{2}, k_{2}+k_{3}, k_{3}+k_{1}\right\}, \\
& h_{5}=\min \left\{\left(k_{1}+k_{2}\right)^{2},\left(k_{2}+k_{3}\right)^{2},\left(k_{3}+k_{1}\right)^{2}\right\} .
\end{aligned}
$$

Differentiating $\Psi\left(R_{e}\right)$ and $\boldsymbol{e}_{R}\left(R_{e}\right)$ in (14) with respect to time and using $\dot{R}_{e}=R_{e} S\left(\boldsymbol{\omega}_{e}\right)$ lead to

$$
\begin{aligned}
\dot{\Psi}\left(R_{e}\right) & =-\frac{1}{2} \operatorname{tr}\left(K R_{e} S\left(\boldsymbol{\omega}_{e}\right)\right) \\
& =\frac{1}{2} \boldsymbol{\omega}_{e}^{\mathrm{T}}\left(K R_{e}-R_{e}^{\mathrm{T}} K\right)^{\mathrm{V}}=\boldsymbol{e}_{R}^{\mathrm{T}} \boldsymbol{\omega}_{e}, \\
\dot{\boldsymbol{e}}_{R}\left(R_{e}\right)= & \frac{1}{2}\left(K R_{e} S\left(\boldsymbol{\omega}_{e}\right)-S^{\mathrm{T}}\left(\boldsymbol{\omega}_{e}\right) R_{e}^{\mathrm{T}} K\right)^{\mathrm{V}} \\
= & \frac{1}{2}\left(K R_{e} S\left(\boldsymbol{\omega}_{e}\right)+S\left(\boldsymbol{\omega}_{e}\right) R_{e}^{\mathrm{T}} K\right)^{\mathrm{V}} \\
= & \frac{1}{2}\left(S\left(\left[\operatorname{tr}\left(R_{e}^{\mathrm{T}} K\right) I_{3}-R_{e}^{\mathrm{T}} K\right] \boldsymbol{\omega}_{e}\right)\right)^{\mathrm{V}} \\
= & \frac{1}{2}\left[\operatorname{tr}\left(R_{e}^{\mathrm{T}} K\right) I_{3}-R_{e}^{\mathrm{T}} K\right] \boldsymbol{\omega}_{e} \triangleq E\left(R_{e}\right) \boldsymbol{\omega}_{e},
\end{aligned}
$$

where $E\left(R_{e}\right)$ is bounded by $\left\|E\left(R_{e}\right)\right\| \leq \frac{1}{\sqrt{2}} \operatorname{tr}(K)$.

Substituting $\boldsymbol{\omega}_{e}$ in (10) into (1) and (3) yields the relative attitude dynamics as

$$
J \dot{\boldsymbol{\omega}}_{e}=-S(\boldsymbol{\omega}) J \boldsymbol{\omega}-J\left[R_{e}^{\mathrm{T}} \dot{\boldsymbol{\omega}}_{t}+S(\boldsymbol{\omega}) \boldsymbol{\omega}_{e}\right]+\boldsymbol{\tau}+S(\boldsymbol{\rho}) \boldsymbol{f}+\boldsymbol{d}_{\tau},
$$

where $\dot{\boldsymbol{\omega}}_{t}$ can be derived from (7) and (14) as

$$
\dot{\boldsymbol{\omega}}_{t}=-J_{t}^{-1} S\left(\boldsymbol{\omega}_{t}\right) J_{t} \boldsymbol{\omega}_{t}
$$


Thus relative attitude dynamics (18) can be rewritten as

$$
J \dot{\boldsymbol{\omega}}_{e}=-S(\boldsymbol{\omega}) J \boldsymbol{\omega}-J S(\boldsymbol{\omega}) \boldsymbol{\omega}_{e}+\boldsymbol{n}_{A}+\boldsymbol{\tau}+S(\boldsymbol{\rho}) \boldsymbol{f}+\boldsymbol{d}_{\tau},
$$

where $\boldsymbol{n}_{A}=J R_{e}^{\mathrm{T}} J_{t}^{-1} S\left(\boldsymbol{\omega}_{t}\right) J_{t} \boldsymbol{\omega}_{t}$.

Remark 2 In fact, $\boldsymbol{e}_{R}\left(R_{e}\right)$ asymptotically converges to zero does not imply that $R \rightarrow R_{t}$ as $t \rightarrow \infty$, since there exist three additional non-degenerate critical points of $\Psi\left(R_{e}\right)$ when $\boldsymbol{e}_{R}\left(R_{e}\right)=0$ (all of four critical points of the function $\Psi\left(R_{e}\right)$ are $I_{3}, \operatorname{diag}\{1,-1,-1\}, \operatorname{diag}\{-1,1,-1\}, \operatorname{diag}\{-1,-1,1\}$, but the unique minimum point of $\Psi\left(R_{e}\right)$ is $\left.I_{3}[41]\right)$, this is due to the nonlinear structures of $\mathrm{SO}(3)$, and these cannot be avoided for any continuous attitude control systems[42].

Remark 3 The globally defined mechanical model of relative attitude and relative position for autonomous proximity operations is established by (11), (13), (17) and (19). From the terms $S(\boldsymbol{\omega}) \boldsymbol{r}_{e}$ in (11) and $\boldsymbol{n}_{P}$ in (13), we know the information of relative attitude motion is involved in the relative position motion. Thus the relative position is significantly affected by the relative attitude between two spacecraft. This reflects that the spacecraft proximity systems have inherently kinematic couplings.

Assumption $1 J$ is an unknown symmetric positive definite constant matrix and bounded by $\lambda_{m} \leq\|J\| \leq \lambda_{M}$ with known constants $\lambda_{m}$ and $\lambda_{M} ; m$ is an unknown positive constant; $\rho$ is an unknown constant vector and bounded by $\|\rho\| \leq \bar{\rho}$ with an unknown constant $\bar{\rho} ; J_{t}$ is an unknown symmetric positive definite constant matrix, and it is bounded by $\left\|J_{t}^{-1}\right\|\left\|J_{t}\right\| \leq \lambda_{t}$ with an unknown constant $\lambda_{t} ; \boldsymbol{d}_{f}$ and $\boldsymbol{d}_{\tau}$ are unknown vectors and bounded by $\left\|\boldsymbol{d}_{f}\right\| \leq \rho_{f}$ and $\left\|\boldsymbol{d}_{\tau}\right\| \leq \rho_{\tau}$ with unknown constants $\rho_{f}$ and $\rho_{\tau}$, respectively.

Assumption 2 The circular-orbit radius $r_{o}$ of the target is known in advance for the chaser. The chaser's motion information $\{R, \boldsymbol{\omega}, \boldsymbol{r}, \boldsymbol{v}\}$ and relative motion information $\left\{R_{e}, \boldsymbol{\omega}_{e}, \boldsymbol{r}_{e}, \boldsymbol{v}_{e}\right\}$ can be directly obtained by the measurement devices mounted on the chaser body[43][44]. However, the target's motion information $\left\{R_{t}, \boldsymbol{\omega}_{t}, \boldsymbol{r}_{t}, \boldsymbol{v}_{t}\right\}$ are assumed to be unmeasurable directly by the chaser. 


\subsection{Control Objective}

The objective in this study is to design controllers based on the proposed model to drive the chaser spacecraft at a desired position $\boldsymbol{p}_{t}$ along the docking port direction of the space target and reorient the chaser's attitude synchronized with the tumbling target attitude. In view of (10) and (14), the objective is equivalent to design effective control inputs $\boldsymbol{f}$ and $\boldsymbol{\tau}$ under Assumptions 1 and 2 , such that $\boldsymbol{r}_{e}$ and $\boldsymbol{e}_{R}$ converge to sufficiently small neighborhood of zero.

\section{Robust Adaptive Controller Design}

Robust adaptive control deals with redesigning or modifying adaptive control schemes to make them robust with respect to bounded disturbances. In this section, robust adaptive controllers are developed for the relative position and relative attitude in spacecraft proximity maneuvers. The adaptive control laws are used to compensate the parametric uncertainties. The robustness is achieved at the expense of replacing the asymptotic tracking property of the dynamics model without kinematic couplings and model uncertainties.

\subsection{Relative Position Controller}

Before developing the relative position controller, we can derive $\left\|\boldsymbol{d}_{f}\right\| \leq$ $\rho_{f}$ from Assumption 1 and $\left\|\boldsymbol{\omega}_{t}\right\|^{2} \leq \frac{2 E_{n}(0)}{\lambda_{J_{t}}}$ from Remark 1, where $\lambda_{J_{t}}$ is the minimum eigenvalue of $J_{t}$, thus

$$
\begin{aligned}
\left\|\boldsymbol{d}_{f}-\boldsymbol{n}_{P}\right\| & \leq\left\|\boldsymbol{d}_{f}\right\|+m\left\|R_{e}^{\mathrm{T}}\right\|\left\|S\left(\boldsymbol{p}_{t}\right)\right\|\left\|\boldsymbol{\omega}_{t}\right\|^{2}\left\|J_{t}^{-1}\right\|\left\|J_{t}\right\| \\
& =\left\|\boldsymbol{d}_{f}\right\|+m\left\|\boldsymbol{p}_{t}\right\|\left\|\boldsymbol{\omega}_{t}\right\|^{2}\left\|J_{t}^{-1}\right\|\left\|J_{t}\right\| \\
& \leq \frac{2 E_{n}(0) m \lambda_{t}}{\lambda_{J_{t}}}+\rho_{f} \triangleq \delta_{P} .
\end{aligned}
$$

where $\left\|R_{e}\right\|=\left\|R_{e}^{\mathrm{T}}\right\|=1$ and $\left\|S\left(\boldsymbol{p}_{t}\right)\right\|=\left\|\boldsymbol{p}_{t}\right\|$. Relative position control system requires the knowledge of mass $m$ and the bound $\delta_{P}$, but it is difficult to measure the values of mass $m$ and the bound $\delta_{P}$ exactly. In general, there are estimation errors:

$$
\tilde{m}=m-\hat{m}, \quad \tilde{\delta}_{P}=\delta_{P}-\hat{\delta}_{P},
$$


where the exact mass and its estimate are denoted by $m$ and $\hat{m}, \hat{\delta}_{P}$ is the estimate of $\delta_{P}$. Then, a robust adaptive relative position controller is designed in Theorem 1.

Theorem 1 Consider the relative position model (11) and (13) in close proximity operations under Assumptions 1 and 2, if the relative position controller is designed by

$$
\begin{gathered}
\boldsymbol{f}=-k_{r} \boldsymbol{r}_{e}-k_{v} \boldsymbol{e}_{P}+\hat{m} \boldsymbol{g}+\boldsymbol{\nu}_{P}, \\
\boldsymbol{\nu}_{P}=-\hat{\delta}_{P} \tanh \left(\gamma \boldsymbol{e}_{P}\right),
\end{gathered}
$$

and corresponding parameter adaptive updating law is assigned as

$$
\dot{\hat{m}}=-k_{m}\left(\boldsymbol{e}_{P}^{\mathrm{T}} \boldsymbol{g}+\sigma_{1} \hat{m}\right),
$$

220

$$
\dot{\hat{\delta}}_{P}=-k_{P}\left[\boldsymbol{e}_{P}^{\mathrm{T}} \tanh \left(\gamma \boldsymbol{e}_{P}\right)+\sigma_{1} \hat{\delta}_{P}\right]
$$

where $\boldsymbol{e}_{P} \in \mathbb{R}^{3}$ is an augmented error vector given by

$$
\boldsymbol{e}_{P}=\boldsymbol{v}_{e}+c_{1} \boldsymbol{r}_{e}
$$

and $k_{r}>0, k_{v}>0, k_{m}>0, k_{P}>0, c_{1}>0, \sigma_{1}>0, \gamma>0$. Then estimation errors $\tilde{m}, \tilde{\delta}_{P}$ are bounded and relative position $\boldsymbol{r}_{e}$ converges to the sufficiently small neighborhood of zero.

Proof Consider a Lyapunov candidate

$$
V_{1}=\frac{1}{2} k_{r} \boldsymbol{r}_{e}^{\mathrm{T}} \boldsymbol{r}_{e}+\frac{1}{2} m \boldsymbol{e}_{P}^{\mathrm{T}} \boldsymbol{e}_{P}+\frac{1}{2 k_{m}} \tilde{m}^{2}+\frac{1}{2 k_{P}} \tilde{\delta}_{P}^{2}
$$

Then, we obtain $V_{1}=\boldsymbol{z}_{1}^{\mathrm{T}} U_{1} \boldsymbol{z}_{1}+\frac{1}{2 k_{m}} \tilde{m}^{2}+\frac{1}{2 k_{P}} \tilde{\delta}_{P}^{2}$ and

$$
\mu_{1}\left\|\boldsymbol{z}_{1}\right\|^{2} \leq \boldsymbol{z}_{1}^{\mathrm{T}} U_{1} \boldsymbol{z}_{1} \leq \mu_{2}\left\|\boldsymbol{z}_{1}\right\|^{2}
$$

where $\boldsymbol{z}_{1}=\left[\left\|\boldsymbol{r}_{e}\right\|,\left\|\boldsymbol{e}_{P}\right\|\right]^{\mathrm{T}} \in \mathbb{R}^{2} ; U_{1}=\frac{1}{2} \operatorname{diag}\left\{k_{r}, m\right\} \in \mathbb{R}^{2 \times 2} ; \mu_{1}$ and $\mu_{2}$ are the minimum eigenvalue and the maximum eigenvalue of matrix $U_{1}$, respectively.

Substituting (22) into (13) leads to the closed-loop position system

$$
m \dot{\boldsymbol{v}}_{e}=-k_{r} \boldsymbol{r}_{e}-k_{v} \boldsymbol{e}_{P}-\tilde{m} \boldsymbol{g}+\boldsymbol{\nu}_{P}-\boldsymbol{n}_{P}+\boldsymbol{d}_{f}
$$


Using $\dot{\tilde{m}}=-\dot{\hat{m}}, \dot{\tilde{\delta}}_{P}=-\dot{\hat{\delta}}_{P}, \boldsymbol{r}_{e}^{\mathrm{T}} S(\boldsymbol{\omega}) \boldsymbol{r}_{e}=0,(11),(26)$, and (29), the timederivative of $V_{1}$ is given by

$$
\begin{aligned}
\dot{V}_{1}= & -k_{r} c_{2}\left\|\boldsymbol{r}_{e}\right\|^{2}-k_{v}\left\|\boldsymbol{e}_{P}\right\|^{2}-\tilde{m} \boldsymbol{e}_{P}^{\mathrm{T}} \boldsymbol{g}+\frac{1}{k_{m}} \tilde{m} \dot{\tilde{m}} \\
& +\frac{1}{k_{P}} \tilde{\delta}_{P} \dot{\tilde{\delta}}_{P}+\boldsymbol{e}_{P}^{\mathrm{T}}\left(\boldsymbol{d}_{f}-\boldsymbol{n}_{P}+\boldsymbol{\nu}_{P}\right) \\
= & -k_{r} c_{1}\left\|\boldsymbol{r}_{e}\right\|^{2}-k_{v}\left\|\boldsymbol{e}_{P}\right\|^{2}+\sigma_{1} \tilde{m} \hat{m}+\sigma_{1} \tilde{\delta}_{P} \hat{\delta}_{P} \\
& -\tilde{\delta}_{P} \boldsymbol{e}_{P}^{\mathrm{T}} \tanh \left(\gamma \boldsymbol{e}_{P}\right)+\boldsymbol{e}_{P}^{\mathrm{T}}\left(\boldsymbol{d}_{f}-\boldsymbol{n}_{P}+\boldsymbol{\nu}_{P}\right) .
\end{aligned}
$$

Since

$$
\begin{aligned}
& \boldsymbol{e}_{P}^{\mathrm{T}}\left(\boldsymbol{d}_{f}-\boldsymbol{n}_{P}+\boldsymbol{\nu}_{P}\right) \leq \delta_{P}\left\|\boldsymbol{e}_{P}\right\|_{1}-\hat{\delta}_{P} \boldsymbol{e}_{P}^{\mathrm{T}} \tanh \left(\gamma \boldsymbol{e}_{P}\right) \\
= & \delta_{P}\left[\sum_{i=1}^{3}\left|e_{P_{i}}\right|-e_{P_{i}} \tanh \left(\gamma e_{P_{i}}\right)\right]+\tilde{\delta}_{P} \boldsymbol{e}_{P}^{\mathrm{T}} \tanh \left(\gamma \boldsymbol{e}_{P}\right) \\
\leq & \frac{\epsilon \delta_{P}}{3 \gamma}+\tilde{\delta}_{P} \boldsymbol{e}_{P}^{\mathrm{T}} \tanh \left(\gamma \boldsymbol{e}_{P}\right),
\end{aligned}
$$

230

$$
\begin{gathered}
\tilde{m} \hat{m}=\tilde{m}(m-\tilde{m}) \leq-\frac{1}{2} \tilde{m}^{2}+\frac{1}{2} m^{2}, \\
\tilde{\delta}_{P} \hat{\delta}_{P}=\tilde{\delta}_{P}\left(\delta_{P}-\tilde{\delta}_{P}\right) \leq-\frac{1}{2} \tilde{\delta}_{P}^{2}+\frac{1}{2} \delta_{P}^{2},
\end{gathered}
$$

then substituting (31)-(33) into (30) gives rise to

$$
\dot{V}_{1} \leq-\boldsymbol{z}_{1}^{\mathrm{T}} U_{2} \boldsymbol{z}_{1}+\kappa_{1}
$$

where $U_{2}=\operatorname{diag}\left\{k_{r} c_{1}, k_{v}\right\}, \kappa_{1}=\frac{1}{2} \sigma_{1}\left(m^{2}+\delta_{P}^{2}\right)+\frac{\epsilon}{3 \gamma} \delta_{P}$.

Since $k_{r}, k_{v}, k_{m}, c_{1}, \sigma_{1}$ are positive constants, then $U_{1}, U_{2}$ are positive defi235 nite. Now, we have

$$
\dot{V}_{1} \leq-\frac{\mu_{3}}{\mu_{2}} V_{1}+\kappa_{2}
$$

where $\mu_{3}$ is the minimum eigenvalue of matrix $U_{2}$ and $\kappa_{2}=\kappa_{1}+\frac{\mu_{3}}{\mu_{2}}\left(\frac{1}{2 k_{m}} \tilde{m}^{2}+\right.$ $\left.\frac{1}{2 k_{P}} \tilde{\delta}_{P}^{2}\right)$. This implies that

$$
V_{1}(t) \leq\left(V_{1}(0)-\frac{\mu_{2} \kappa_{2}}{\mu_{3}}\right) \mathrm{e}^{-\frac{\mu_{3}}{\mu_{2}} t}+\frac{\mu_{2} \kappa_{2}}{\mu_{3}} .
$$

This means $\boldsymbol{r}_{e}, \boldsymbol{e}_{P}, \tilde{m}, \tilde{\delta}_{P}$ are bounded. Then from (28), we have

$$
\left\|\boldsymbol{r}_{e}(t)\right\| \leq\left\|\boldsymbol{z}_{1}(t)\right\| \leq \sqrt{\frac{1}{\mu_{1}}\left(V_{1}(0)-\frac{\mu_{2} \kappa_{2}}{\mu_{3}}\right) \mathrm{e}^{-\frac{\mu_{3}}{\mu_{2}} t}+\frac{\mu_{2} \kappa_{2}}{\mu_{1} \mu_{3}}} .
$$


Thus, $\lim _{t \rightarrow \infty}\left\|\boldsymbol{r}_{e}(t)\right\| \leq \sqrt{\frac{\mu_{2} \kappa_{2}}{\mu_{1} \mu_{3}}}$, and the ultimately uniformly boundedness of the relative position is proved. Moreover, smaller $\sigma_{1}$ and larger $c_{1}, k_{v}, \gamma, k_{m}, k_{P}$ result in larger $\mu_{3}$ and smaller $\kappa_{2}$, thus the sufficiently small ultimate bound of $\left\|\boldsymbol{r}_{e}(\infty)\right\|$ can be regulated.

\subsection{Relative Attitude Controller}

Before developing the relative attitude controller, we also can derive $\left\|\boldsymbol{d}_{\tau}\right\| \leq$ $\rho_{\tau}$ from Assumption 1 and $\left\|\boldsymbol{\omega}_{t}\right\|^{2} \leq \frac{2 E_{n}(0)}{\lambda_{J_{t}}}$ from Remark 1, thus

$$
\begin{aligned}
\left\|\boldsymbol{n}_{A}+\boldsymbol{d}_{\tau}\right\| & \leq\|J\|\left\|R_{e}^{\mathrm{T}}\right\|\left\|\boldsymbol{\omega}_{t}\right\|^{2}\left\|J_{t}^{-1}\right\|\left\|J_{t}\right\|+\left\|\boldsymbol{d}_{\tau}\right\| \\
& =\|J\|\left\|\boldsymbol{\omega}_{t}\right\|^{2}\left\|J_{t}^{-1}\right\|\left\|J_{t}\right\|+\left\|\boldsymbol{d}_{\tau}\right\| \\
& \leq \frac{2 E_{n}(0) \lambda_{M} \lambda_{t}}{\lambda_{J_{t}}}+\rho_{\tau} \triangleq \delta_{A}
\end{aligned}
$$

Relative attitude control system requires the knowledge of an inertia matrix $J$, thrust misalignment vector $\rho$ and the bound $\delta_{A}$, but it is difficult to measure them exactly. In general, there are estimation errors:

$$
\tilde{J}=J-\hat{J}, \quad \tilde{\boldsymbol{\rho}}=\boldsymbol{\rho}-\hat{\boldsymbol{\rho}}, \quad \tilde{\delta}_{A}=\delta_{A}-\hat{\delta}_{A},
$$

where the exact inertia matrix and its estimate are denoted by $J$ and $\hat{J}$, all of matrices $J, \hat{J}, \tilde{J}$ are symmetric; the exact thrust misalignment vector and its estimate are denoted by $\boldsymbol{\rho}$ and $\hat{\boldsymbol{\rho}} ; \hat{\delta}_{A}$ is the estimate of $\delta_{A}$. Then, inspired by the single rigid body attitude tracking controller in [40], a robust adaptive controller can be designed for relative attitude in Theorem 2 .

Theorem 2 Consider the relative attitude model (17) and (19) for close proximity operations under Assumptions 1 and 2, if the adaptive relative attitude controller is designed by

$$
\boldsymbol{\tau}=-k_{R} \boldsymbol{e}_{R}-k_{w} \boldsymbol{\omega}_{e}+S(\boldsymbol{\omega}) \hat{J} \boldsymbol{\omega}+\hat{J} S(\boldsymbol{\omega}) \boldsymbol{\omega}_{e}-S(\hat{\boldsymbol{\rho}}) \boldsymbol{f}+\boldsymbol{\nu}_{A},
$$

$$
\boldsymbol{\nu}_{A}=-\hat{\delta}_{A} \tanh \left(\gamma \boldsymbol{e}_{A}\right)
$$


and corresponding parameter adaptive updating law is assigned as

$$
\begin{gathered}
\dot{\hat{J}}=\frac{k_{J}}{2}\left[-S(\boldsymbol{\omega}) \boldsymbol{\omega}_{e} \boldsymbol{e}_{A}^{\mathrm{T}}-\boldsymbol{e}_{A}\left(S(\boldsymbol{\omega}) \boldsymbol{\omega}_{e}\right)^{\mathrm{T}}\right. \\
\left.+\boldsymbol{\omega} \boldsymbol{\omega}^{\mathrm{T}} S\left(\boldsymbol{e}_{A}\right)-S\left(\boldsymbol{e}_{A}\right) \boldsymbol{\omega} \boldsymbol{\omega}^{\mathrm{T}}-2 \sigma_{2} \hat{J}\right], \\
\dot{\hat{\boldsymbol{\rho}}}=-k_{\rho}\left[S^{\mathrm{T}}(\boldsymbol{f}) \boldsymbol{e}_{A}+\sigma_{2} \hat{\boldsymbol{\rho}}\right], \\
\dot{\hat{\delta}}_{A}=-k_{A}\left[\boldsymbol{e}_{A}^{\mathrm{T}} \tanh \left(\gamma \boldsymbol{e}_{A}\right)+\sigma_{2} \hat{\delta}_{A}\right],
\end{gathered}
$$

where $\boldsymbol{e}_{A} \in \mathbb{R}^{3}$ is an augmented error vector given by

$$
e_{A}=\omega_{e}+c_{2} e_{R}
$$

for a constant $c_{2}$ satisfying

$$
0<c_{2}<\min \left\{\sqrt{\frac{2 b_{1} k_{R} \lambda_{m}}{\lambda_{M}^{2}}}, \frac{\sqrt{2} k_{w}}{\lambda_{M} \operatorname{tr}(K)}, \frac{4 k_{R} k_{w}}{k_{w}^{2}+\frac{1}{\sqrt{2}} k_{R} \lambda_{M} \operatorname{tr}(K)}\right\} .
$$

260

and $k_{R}>0, k_{w}>0, k_{J}>0, k_{A}>0, k_{\rho}>0, \sigma_{2}>0, \gamma>0$. Then estimation errors $\tilde{J}, \tilde{\boldsymbol{\rho}}, \tilde{\delta}_{A}$ are bounded and relative attitude $\boldsymbol{e}_{R}$ converges to the sufficiently small neighborhood of zero.

Proof Consider a Lyapunov candidate

$$
\begin{aligned}
V_{2}= & \frac{1}{2} \boldsymbol{\omega}_{e}^{\mathrm{T}} J \boldsymbol{\omega}_{e}+k_{R} \Psi\left(R_{e}\right)+c_{1} \boldsymbol{\omega}_{e}^{\mathrm{T}} J \boldsymbol{e}_{R} \\
& +\frac{1}{2 k_{J}}\|\tilde{J}\|_{F}^{2}+\frac{1}{2 k_{\rho}}\|\tilde{\boldsymbol{\rho}}\|^{2}+\frac{1}{2 k_{A}} \tilde{\delta}_{A}^{2} .
\end{aligned}
$$

For a positive constant $\psi<h_{1}$, define $\mathrm{D} \subset \mathrm{SO}(3)$ as

$$
\mathrm{D} \triangleq\left\{R_{e} \in \mathrm{SO}(3): \Psi\left(R_{e}\right)<\psi<h_{1}\right\}
$$

From Assumption 1, $V_{2}$ is bounded in $\mathrm{D}$ by

$$
\boldsymbol{z}_{2}^{\mathrm{T}} W_{1} \boldsymbol{z}_{2} \leq V_{2} \leq \boldsymbol{z}_{2}^{\mathrm{T}} W_{2} \boldsymbol{z}_{2}+\frac{1}{2 k_{J}}\|\tilde{J}\|_{F}^{2}+\frac{1}{2 k_{\rho}}\|\tilde{\boldsymbol{\rho}}\|^{2}+\frac{1}{2 k_{A}} \tilde{\delta}_{A}^{2},
$$

where $\boldsymbol{z}_{2}=\left[\left\|\boldsymbol{e}_{R}\right\|,\left\|\boldsymbol{\omega}_{e}\right\|\right]^{\mathrm{T}} \in \mathbb{R}^{2}$, and the matrices $W_{1}, W_{2} \in \mathbb{R}^{2 \times 2}$ are given by

$$
W_{1}=\left[\begin{array}{cc}
b_{1} k_{R} & \frac{1}{2} c_{2} \lambda_{M} \\
\frac{1}{2} c_{2} \lambda_{M} & \frac{1}{2} \lambda_{m}
\end{array}\right]
$$




$$
W_{2}=\left[\begin{array}{cc}
b_{2} k_{R} & \frac{1}{2} c_{2} \lambda_{M} \\
\frac{1}{2} c_{2} \lambda_{M} & \frac{1}{2} \lambda_{M}
\end{array}\right]
$$

Substituting (40) into (19) leads to the closed-loop attitude system

$$
\begin{aligned}
J \dot{\boldsymbol{\omega}}_{e}= & -k_{R} \boldsymbol{e}_{R}-k_{w} \boldsymbol{\omega}_{e}-S(\boldsymbol{\omega}) \tilde{J} \boldsymbol{\omega}-\tilde{J} S(\boldsymbol{\omega}) \boldsymbol{\omega}_{e} \\
& +S(\tilde{\boldsymbol{\rho}}) \boldsymbol{f}+\boldsymbol{\nu}_{A}+\boldsymbol{n}_{A}+\boldsymbol{d}_{\tau} .
\end{aligned}
$$

Using (16), (17) and (49), the time-derivative of $V_{2}$ is given by

$$
\begin{aligned}
\dot{V}_{2}= & -k_{w}\left\|\boldsymbol{\omega}_{e}\right\|^{2}-c_{2} k_{R}\left\|\boldsymbol{e}_{R}\right\|^{2}+c_{2} \boldsymbol{\omega}_{e}^{\mathrm{T}} J E \boldsymbol{\omega}_{e}-c_{2} k_{w} \boldsymbol{\omega}_{e}^{\mathrm{T}} \boldsymbol{e}_{R} \\
& -\boldsymbol{e}_{A}^{\mathrm{T}}\left[S(\boldsymbol{\omega}) \tilde{J} \boldsymbol{\omega}+\tilde{J} S(\boldsymbol{\omega}) \boldsymbol{\omega}_{e}\right]+\boldsymbol{e}_{A}^{\mathrm{T}} S(\tilde{\boldsymbol{\rho}}) \boldsymbol{f}+\frac{1}{k_{J}} \operatorname{tr}(\tilde{J} \dot{\tilde{J}}) \\
& +\frac{1}{k_{\rho}} \tilde{\boldsymbol{\rho}}^{\mathrm{T}} \dot{\tilde{\boldsymbol{\rho}}}+\frac{1}{k_{J}} \tilde{\delta}_{A} \dot{\tilde{\delta}}_{A}+\boldsymbol{e}_{A}^{\mathrm{T}}\left(\boldsymbol{\nu}_{A}+\boldsymbol{n}_{A}+\boldsymbol{d}_{\tau}\right) .
\end{aligned}
$$

Using the following facts that $\boldsymbol{x}^{\mathrm{T}} \boldsymbol{y}=\operatorname{tr}\left(\boldsymbol{x} \boldsymbol{y}^{\mathrm{T}}\right)=\operatorname{tr}\left(\boldsymbol{y} \boldsymbol{x}^{\mathrm{T}}\right)$ and $\operatorname{tr}(\tilde{J} A)=\operatorname{tr}\left(\tilde{J} A^{\mathrm{T}}\right)$ for any $\boldsymbol{x}, \boldsymbol{y} \in \mathbb{R}^{3}$ and $A \in \mathbb{R}^{3 \times 3} ;\left(S\left(\boldsymbol{e}_{A}\right) \boldsymbol{\omega}\right)^{\mathrm{T}}=-\boldsymbol{\omega}^{\mathrm{T}} S\left(\boldsymbol{e}_{A}\right) ; S(\tilde{\boldsymbol{\rho}}) \boldsymbol{f}=-S(\boldsymbol{f}) \tilde{\boldsymbol{\rho}}$; $\dot{\tilde{J}}=-\dot{\hat{J}}, \dot{\tilde{\boldsymbol{\rho}}}=-\dot{\hat{\boldsymbol{\rho}}}$ and $\dot{\tilde{\delta}}_{A}=-\dot{\hat{\delta}}_{A}$, then we get

$$
\begin{aligned}
\dot{V}_{2}= & -k_{w}\left\|\boldsymbol{\omega}_{e}\right\|^{2}-c_{2} k_{R}\left\|\boldsymbol{e}_{R}\right\|^{2}+c_{2} \boldsymbol{\omega}_{e}^{\mathrm{T}} J E \boldsymbol{\omega}_{e}-c_{2} k_{w} \boldsymbol{\omega}_{e}^{\mathrm{T}} \boldsymbol{e}_{R} \\
& +\operatorname{tr}\left(\tilde{J}\left[-S(\boldsymbol{\omega}) \boldsymbol{\omega}_{e} \boldsymbol{e}_{A}^{\mathrm{T}}-\boldsymbol{\omega}\left(S\left(\boldsymbol{e}_{A}\right) \boldsymbol{\omega}\right)^{\mathrm{T}}+\frac{1}{k_{J}} \dot{\tilde{J}}\right]\right) \\
& -\tilde{\boldsymbol{\rho}}^{\mathrm{T}}\left[S^{\mathrm{T}}(\boldsymbol{f}) \boldsymbol{e}_{A}-\frac{1}{k_{\rho}} \dot{\tilde{\boldsymbol{\rho}}}\right]-\tilde{\delta}_{A} \boldsymbol{e}_{A}^{\mathrm{T}} \tanh \left(\gamma \boldsymbol{e}_{A}\right) \\
& +\sigma_{2} \tilde{\delta}_{A} \hat{\delta}_{A}+\boldsymbol{e}_{A}^{\mathrm{T}}\left(\boldsymbol{\nu}_{A}+\boldsymbol{n}_{A}+\boldsymbol{d}_{\tau}\right) \\
= & -k_{w}\left\|\boldsymbol{\omega}_{e}\right\|^{2}-c_{2} k_{R}\left\|\boldsymbol{e}_{R}\right\|^{2}+c_{2} \boldsymbol{\omega}_{e}^{\mathrm{T}} J E \boldsymbol{\omega}_{e}-c_{2} k_{w} \boldsymbol{\omega}_{e}^{\mathrm{T}} \boldsymbol{e}_{R} \\
& +\sigma_{2} \operatorname{tr}(\tilde{J} \hat{J})+\sigma_{2} \tilde{\boldsymbol{\rho}}^{\mathrm{T}} \hat{\boldsymbol{\rho}}-\tilde{\delta}_{A} \boldsymbol{e}_{A}^{\mathrm{T}} \tanh \left(\gamma \boldsymbol{e}_{A}\right) \\
& +\sigma_{2} \tilde{\delta}_{A} \hat{\delta}_{A}+\boldsymbol{e}_{A}^{\mathrm{T}}\left(\boldsymbol{\nu}_{A}+\boldsymbol{n}_{A}+\boldsymbol{d}_{\tau}\right) .
\end{aligned}
$$

Since $\|J\|_{F} \leq \sqrt{3}\|J\| \leq \sqrt{3} \lambda_{M}$

$$
\begin{aligned}
& \boldsymbol{e}_{A}^{\mathrm{T}}\left(\boldsymbol{n}_{A}+\boldsymbol{d}_{\tau}+\boldsymbol{\nu}_{A}\right) \leq \delta_{A}\left\|\boldsymbol{e}_{A}\right\|_{1}-\hat{\delta}_{A} \boldsymbol{e}_{A}^{\mathrm{T}} \tanh \left(\gamma \boldsymbol{e}_{A}\right) \\
= & \delta_{A}\left[\sum_{i=1}^{3}\left|e_{A_{i}}\right|-e_{A_{i}} \tanh \left(\gamma e_{A_{i}}\right)\right]+\tilde{\delta}_{A} \boldsymbol{e}_{A}^{\mathrm{T}} \tanh \left(\gamma \boldsymbol{e}_{A}\right) \\
\leq & \frac{\epsilon \delta_{A}}{3 \gamma}+\tilde{\delta}_{A} \boldsymbol{e}_{A}^{\mathrm{T}} \tanh \left(\gamma \boldsymbol{e}_{A}\right),
\end{aligned}
$$




$$
\begin{aligned}
& \operatorname{tr}(\tilde{J} \hat{J})=\operatorname{tr}(\tilde{J}(J-\tilde{J}))=\sum_{i=1}^{3}\left(J_{i i} \tilde{J}_{i i}-\tilde{J}_{i i}^{2}\right) \\
& \leq \sum_{i=1}^{3}\left(-\frac{1}{2} \tilde{J}_{i i}^{2}+\frac{1}{2} J_{i i}^{2}\right)=-\frac{1}{2}\|\tilde{J}\|_{F}^{2}+\frac{1}{2}\|J\|_{F}^{2} \\
& \leq-\frac{1}{2}\|\tilde{J}\|_{F}^{2}+\frac{3}{2} \lambda_{M}^{2}, \\
& \tilde{\boldsymbol{\rho}}^{\mathrm{T}} \hat{\boldsymbol{\rho}}=\tilde{\boldsymbol{\rho}}^{\mathrm{T}}(\boldsymbol{\rho}-\tilde{\boldsymbol{\rho}}) \leq-\frac{1}{2}\|\tilde{\boldsymbol{\rho}}\|^{2}+\frac{1}{2}\|\boldsymbol{\rho}\|^{2} \leq-\frac{1}{2}\|\tilde{\boldsymbol{\rho}}\|^{2}+\frac{1}{2} \bar{\rho}^{2},
\end{aligned}
$$

265

$$
\tilde{\delta}_{A} \hat{\delta}_{A}=\tilde{\delta}_{A}\left(\delta_{A}-\tilde{\delta}_{A}\right) \leq-\frac{1}{2} \tilde{\delta}_{A}^{2}+\frac{1}{2} \delta_{A}^{2},
$$

then substituting (52)-(55) into (51) gives rise to

$$
\dot{V}_{2} \leq-z_{2}^{\mathrm{T}} W_{3} z_{2}+\kappa_{3}
$$

where $\kappa_{3}=\frac{1}{2} \sigma_{2}\left(3 \lambda_{M}^{2}+\bar{\rho}^{2}+\delta_{A}^{2}\right)+\frac{\epsilon}{3 \gamma} \delta_{A}$,

$$
W_{3}=\left[\begin{array}{cc}
c_{2} k_{R} & -\frac{1}{2} c_{2} k_{w} \\
-\frac{1}{2} c_{2} k_{w} & k_{w}-\frac{1}{\sqrt{2}} c_{2} \lambda_{M} \operatorname{tr}(K)
\end{array}\right] .
$$

The inequality (46) for the constant $c_{2}$ guarantees that the matrices $W_{1}, W_{2}, W_{3}$ become positive definite. Then, we have

$$
\dot{V}_{2} \leq-\frac{\lambda_{3}}{\lambda_{2}} V_{2}+\kappa_{4}
$$

where $\lambda_{3}$ and $\lambda_{2}$ represent the minimum eigenvalue and the maximum eigenvalue of matrices $W_{3}$ and $W_{2}$, respectively; $\kappa_{4}=\kappa_{3}+\frac{\lambda_{3}}{\lambda_{2}}\left(\frac{1}{2 k_{J}}\|\tilde{J}\|_{F}^{2}+\frac{1}{2 k_{\rho}}\|\tilde{\boldsymbol{\rho}}\|^{2}+\frac{1}{2 k_{A}} \tilde{\delta}_{A}^{2}\right)$. Thus $\dot{V}_{2}(t)<0$ when $V_{2}(t)>\frac{\lambda_{2} \kappa_{1}}{\lambda_{3}}$, and

$$
V_{2}(t) \leq\left(V_{2}(0)-\frac{\lambda_{2} \kappa_{4}}{\lambda_{3}}\right) \mathrm{e}^{-\frac{\lambda_{3}}{\lambda_{2}} t}+\frac{\lambda_{2} \kappa_{4}}{\lambda_{3}} .
$$

This means $\boldsymbol{e}_{R}(t), \boldsymbol{\omega}_{e}, \tilde{J}, \tilde{\boldsymbol{\rho}}, \tilde{\delta}_{A}$ are bounded. Furthermore, from (48), we have

$$
\left\|\boldsymbol{e}_{R}(t)\right\| \leq\left\|\boldsymbol{z}_{2}(t)\right\| \leq \sqrt{\frac{1}{\lambda_{1}}\left(V_{2}(0)-\frac{\lambda_{2} \kappa_{4}}{\lambda_{3}}\right) \mathrm{e}^{-\frac{\lambda_{3}}{\lambda_{2}} t}+\frac{\lambda_{2} \kappa_{4}}{\lambda_{1} \lambda_{3}}} .
$$

where $\lambda_{1}$ is the minimum eigenvalue of matrix $W_{1}$. 
Let a sublevel set of $V_{2}$ be $\mathrm{V}_{a} \triangleq\left\{\left(R_{e}, \boldsymbol{\omega}_{e}, \hat{J}, \hat{\boldsymbol{\rho}}, \hat{\delta}_{A}\right) \in \mathrm{SO}(3) \times \mathbb{R}^{3} \times \mathbb{R}^{3 \times 3} \times \mathbb{R}^{3} \times\right.$ $\left.\mathbb{R}: V_{2}<\psi_{a}\right\}$ for a constant $\psi_{a}>0$. If the following inequality for $\psi_{a}$ is satisfied $\psi_{a}<\frac{\psi}{b_{2}} \lambda_{1} \triangleq \kappa_{a}$, then we can guarantee that $\mathrm{V}_{a} \subset \mathrm{D} \times \mathbb{R}^{3} \times \mathbb{R}^{3 \times 3} \times \mathbb{R}^{3} \times \mathbb{R}$, since it implies that $\left\|\boldsymbol{z}_{2}\right\|^{2}<\frac{\psi}{b_{2}}$, which leads $\Psi\left(R_{e}\right) \leq b_{2}\left\|\boldsymbol{e}_{R}\right\|^{2} \leq b_{2}\left\|\boldsymbol{z}_{2}\right\|^{2}<\psi$. Thus, from (57), a sublevel set of $\mathrm{V}_{a}$ is a positively invariant set, when $\kappa_{4}<\psi_{a}<\kappa_{a}$, and it becomes smaller until $\psi_{a}=\kappa_{4}$. In order to guarantee the existence of such $\mathrm{V}_{a}$, the following inequality should be satisfied

$$
\kappa_{4}<\frac{\psi}{b_{2}} \lambda_{1}=\kappa_{a}
$$

which can be achieved by choosing sufficiently small $\sigma_{2}$ and large $\gamma, k_{J}, k_{\rho}, k_{A}$. Thus, for any initial condition satisfying $V_{2}(0)<\kappa_{a}$, the relative attitude ultimately converges to the set $\lim _{t \rightarrow \infty}\left\|\boldsymbol{e}_{R}(t)\right\| \leq \sqrt{\frac{\lambda_{2} \kappa_{1}}{\lambda_{1} \lambda_{3}}}$. Thus the ultimately uniformly boundedness of the relative attitude is proved. Moreover, smaller $\sigma_{2}$ and larger $c_{2}, k_{w}, \gamma, k_{J}, k_{\rho}, k_{A}$ result in larger $\lambda_{3}$ and smaller $\kappa_{4}$, thus the sufficiently small ultimate bound of $\left\|\boldsymbol{r}_{e}(\infty)\right\|$ can be regulated.

Remark 4 Outside the set $\mathrm{C} \triangleq\left\{\boldsymbol{z}_{2}: \lambda_{3}\left\|\boldsymbol{z}_{2}\right\|^{2}>\kappa_{4}\right\}$, we have $\dot{V}_{2} \leq 0$ from (57). Inside of the set $\mathrm{B} \triangleq\left\{\boldsymbol{z}_{2}:\left\|\boldsymbol{z}_{2}\right\|^{2} \leq \frac{\psi}{b_{2}} \subset \mathrm{D} \times \mathbb{R}^{3} \times \mathbb{R}^{3 \times 3} \times \mathbb{R}^{3} \times \mathbb{R}\right\}$, equations (48) and (57) hold. The inequality (59) guarantees that the smallest sublevel set $\mathrm{V}_{b}$ of $V_{2}$, covering the set $\mathrm{C}$, lies inside of the largest sublevel set $\mathrm{V}_{c}$ of $V_{2}$ in $\mathrm{B}$, that is to say $\mathrm{V}_{b} \subset \mathrm{V}_{c}$. Therefore, along any solution starting in $\mathrm{V}_{c}, V_{2}$ decreases until the solution enters $\mathrm{V}_{b}$, thereby yielding uniform boundedness. Note that the above aforementioned sets satisfy $C \subset V_{b} \subset V_{c} \subset B \subset D \times \mathbb{R}^{3} \times \mathbb{R}^{3 \times 3} \times \mathbb{R}^{3} \times \mathbb{R}$.

Remark 5 The proposed relative attitude controller (40) is developed based on the rotation matrix rather than the conventional coordinate-dependent design approaches. Since the non-singular range of the euler-angle-based and quaternion-based relative attitude description are $\left(\frac{-\pi}{2}, \frac{\pi}{2}\right)$ and $(-\pi, \pi)$, respectively. Moreover, the modified Rodrigues parameter-based approach should employ the projection and switching mechanisms to achieve the global relative attitude description [45]. The rotation matrix is used to directly design the relative attitude controller can avoid the singularity of the conventional attitude parameters and achieve the almost global relative attitude control. 
Remark 6 It is worthwhile to mention that the proposed robust adaptive controllers (22) and (40) are derived based on the standard $\sigma$-modification scheme [46] to ensure the stability of the estimations, but the convergence of the estimated parameters to their true values cannot be guaranteed, since the persistent excitation conditions are not satisfied [46]. Recently, a new adaptive method was developed in [47] to ensure that the estimated parameters exponentially converge to their true values. This adaptive method was also extended to estimate the unknown neural network weights and sprung mass for the active suspension systems [48] and was used to exactly estimate parameters of robotic systems in finite-time [49]. In spite of the exact estimation of this adaptive method, the regressor matrices should be adapted online such that the computational burden of the controller is also increased as well as the composite adaptive control method in [50].

\subsection{Stability Analysis for Complete System}

Theorem 3 Under Assumption 1 and 2, for the globally defined close proximity operations model, including (11), (13), (17) and (19), if the controllers are defined by (22) and (40) and adaptive laws are assigned by (24), (25), (42) and (43), then closed-loop system states $\boldsymbol{r}_{e}$ and $\boldsymbol{e}_{R}$ converge to sufficiently small neighborhood of zero.

Proof Let $V=V_{1}+V_{2}$ be the Lyapunov candidate of complete system. Using (28) and (48), the bound of $V$ can be given as

$$
\boldsymbol{z}^{\mathrm{T}} M_{1} \boldsymbol{z} \leq V \leq \boldsymbol{z}^{\mathrm{T}} M_{2} \boldsymbol{z}+\vartheta\|\tilde{\boldsymbol{\theta}}\|^{2}
$$

where $\boldsymbol{z} \triangleq\left[\boldsymbol{z}_{1}^{\mathrm{T}}, \boldsymbol{z}_{2}^{\mathrm{T}}\right]^{\mathrm{T}} \in \mathbb{R}^{4}, M_{1}=\operatorname{diag}\left\{U_{1}, W_{1}\right\}, M_{2}=\operatorname{diag}\left\{U_{1}, W_{2}\right\}, \vartheta=$ $\max \left\{\frac{1}{2 k_{m}}, \frac{1}{2 k_{P}}, \frac{1}{2 k_{J}}, \frac{1}{2 k_{\rho}}, \frac{1}{2 k_{A}}\right\}$, and $\tilde{\boldsymbol{\theta}}=\left[\tilde{m}, \tilde{\delta}_{P},\|\tilde{J}\|_{F},\|\tilde{\boldsymbol{\rho}}\|, \tilde{\delta}_{A}\right]^{\mathrm{T}}$.

Using (35) and (51), the time derivative of $V$ is given by

$$
\dot{V} \leq-\boldsymbol{z}^{\mathrm{T}} M_{3} \boldsymbol{z}+\kappa_{0}
$$

where $M_{3}=\operatorname{diag}\left\{U_{2}, W_{3}\right\}, \kappa_{0}=\kappa_{1}+\kappa_{3}$. 
Under the given positive designing parameters, all of the matrices $M_{1}, M_{2}, M_{3}$ are positive definite. Then, we have

$$
\dot{V} \leq-\frac{\eta_{3}}{\eta_{2}} V+\kappa
$$

where $\eta_{3}$ and $\eta_{2}$ represent the minimum eigenvalue and the maximum eigenvalue of matrices $M_{3}$ and $M_{2}$, respectively; $\kappa=\kappa_{0}+\frac{\eta_{3}}{\eta_{2}} \vartheta\|\tilde{\boldsymbol{\theta}}\|^{2}$. Thus we know

$$
V(t) \leq\left(V(0)-\frac{\eta_{2} \kappa}{\eta_{3}}\right) \mathrm{e}^{-\frac{\eta_{3}}{\eta_{2}} t}+\frac{\eta_{2} \kappa}{\eta_{3}} .
$$

This means $\boldsymbol{z}$ and $\tilde{\boldsymbol{\theta}}$ are bounded. Furthermore, from (60), we conclude

$$
\left\|\left[\begin{array}{l}
\boldsymbol{r}_{e}(t) \\
\boldsymbol{e}_{R}(t)
\end{array}\right]\right\| \leq\|\boldsymbol{z}(t)\| \leq \sqrt{\frac{1}{\eta_{1}}\left(V(0)-\frac{\eta_{2} \kappa}{\eta_{3}}\right) \mathrm{e}^{-\frac{\eta_{3}}{\eta_{2}} t}+\frac{\eta_{2} \kappa}{\eta_{1} \eta_{3}}},
$$

where $\eta_{1}$ is the minimum eigenvalue of matrix $M_{1}$. Thus,

$$
\lim _{t \rightarrow \infty}\left\|\left[\boldsymbol{r}_{e}^{\mathrm{T}}(t), \boldsymbol{e}_{R}^{\mathrm{T}}(t)\right]^{\mathrm{T}}\right\| \leq \sqrt{\frac{\eta_{2} \kappa}{\eta_{1} \eta_{3}}},
$$

and smaller $\sigma_{1}, \sigma_{2}$ and larger $c_{1}, c_{2}, k_{v}, k_{w}, \gamma, k_{m}, k_{P}, k_{J}, k_{\rho}, k_{A}$ result in larger $\eta_{3}$ and smaller $\kappa$, thus the sufficiently small ultimate bounds of relative position and relative attitude can be regulated.

Remark 6 Note that the feedback gains $\left\{k_{r}, k_{v}, c_{1}\right\}$ in the relative position controller $(22)$ and $\left\{k_{R}, k_{w}, c_{2}\right\}$ in the relative attitude controller (40) can be chosen independently to satisfy their own bandwidths, respectively.

\section{Numerical Example}

A example of close proximity mission in orbit for rendezvous and docking is simulated in this section. Assume the the chaser's initial position, velocity, attitude, and angular velocity are $\boldsymbol{r}=[1,1,1]^{\mathrm{T}} \times 7.078 \times 10^{8}(\mathrm{~m}), \boldsymbol{v}=$ $[2,3,-2]^{\mathrm{T}}(\mathrm{m} / \mathrm{s}), R(0)=I_{3}$, and $\boldsymbol{\omega}=[0,0,0]^{\mathrm{T}}(\mathrm{rad} / \mathrm{s})$, respectively. The initial relative position, relative velocity, relative attitude, and relative angular velocity are $\boldsymbol{r}_{e}=[50 / \sqrt{2}, 0,-50 / \sqrt{2}]^{\mathrm{T}}(\mathrm{m}), \boldsymbol{v}_{e}=[0.5,-0.5,0.5]^{\mathrm{T}}(\mathrm{m} / \mathrm{s}), R_{e}(0)=I_{3}$, and $\boldsymbol{\omega}_{e}=[0.02,-0.02,0.02]^{\mathrm{T}}(\mathrm{rad} / \mathrm{s})$, respectively. The target's orbital radius is $r_{o}=7.078 \times 10^{8}(\mathrm{~m})$ and the gravitational constant of the Earth is 
$\mu_{g}=3.986 \times 10^{14}\left(\mathrm{~m}^{3} / \mathrm{s}^{2}\right)$. Desired position of the chaser denoted in frame $\mathcal{F}_{t}$ is given as $\boldsymbol{p}_{t}=[0,5,0]^{\mathrm{T}}(\mathrm{m})$. The prior known bounds of the chaser's inertia matrix are $\lambda_{m}=250$ and $\lambda_{M}=610$; the bound of thrust misalignment vector is $\bar{\rho}=0.05$. We use the proposed controllers (22) and (40) to achieve the objective for the chaser approaching a tumbling non-cooperative target. The controller parameters are chosen as $K=0.6 \times \operatorname{diag}\{1,2,3\}, k_{R}=5, k_{w}=60$, $k_{r}=0.5, k_{v}=6, c_{1}=0.1, c_{2}=0.2, k_{J}=k_{m}=0.5, k_{A}=k_{P}=0.2, k_{\rho}=0.2$, $\sigma_{1}=\sigma_{2}=0.01, \gamma=50, \hat{m}(0)=\hat{\delta}_{A}(0)=\hat{\delta}_{P}(0)=0, \hat{\boldsymbol{\rho}}(0)=\mathbf{0}, \hat{J}(0)=O_{3}$.

In the simulation, parameters of the chaser, the target and disturbance are listed as:

$$
\begin{gathered}
\boldsymbol{\rho}=[0.02,0.03,0.025]^{\mathrm{T}}(\mathrm{m}), \quad m=58.2(\mathrm{~kg}), \\
J=\left[\begin{array}{rrr}
598.3 & -22.5 & -51.5 \\
-22.5 & 424.4 & -27 \\
-51.5 & -27 & 263.6
\end{array}\right]\left(\mathrm{kgm}^{2}\right), \\
J_{t}=\left[\begin{array}{lll}
3336.3 & -135.4 & -154.2 \\
-135.4 & 3184.5 & -148.5 \\
-154.2 & -148.5 & 2423.7
\end{array}\right]\left(\mathrm{kgm}^{2}\right), \\
\boldsymbol{d}_{\tau}=\left[\begin{array}{l}
1+7 \sin \left(\omega_{o} t\right)-2 \cos \left(\omega_{o} t\right) \\
8-5 \sin \left(\omega_{o} t\right)+3 \cos \left(\omega_{o} t\right) \\
1+5 \sin \left(\omega_{o} t\right)-2 \cos \left(\omega_{o} t\right)
\end{array}\right] \times 10^{-5}(\mathrm{Nm}), \\
\boldsymbol{d}_{f}=\left[\begin{array}{l}
1-8 \sin \left(\omega_{o} t\right)+2 \cos \left(\omega_{o} t\right) \\
8-6 \sin \left(\omega_{o} t\right)-3 \cos \left(\omega_{o} t\right) \\
1+5 \sin \left(\omega_{o} t\right)+2 \cos \left(\omega_{o} t\right)
\end{array}\right] \times 10^{-4}(\mathrm{~N}) .
\end{gathered}
$$

where $\omega_{o}=\sqrt{\mu_{g} /\|\boldsymbol{r}\|^{3}}$ is the chaser's orbital angular velocity.

Figure 2 shows the time histories of relative position and relative velocity expressed in frame $\mathcal{F}_{c}$. The task of the position control in this simulation is to drive the chaser to a desired position $\boldsymbol{p}_{t}$. The relative position and relative velocity tend to steady state in 150 (s) imply the desired position tracking is achieved. The time history of relative attitude is shown in Figure 3. The 

indicate the chaser attitude is synchronized with the attitude of the target. The control forces and control torques presented in Figure 4 show that the large control efforts of chaser spacecraft are used initially in order to track the desired position and coincide with the tumbling target attitude quickly. Figure 5 and 365 Figure 6 show that the estimation errors of unknown parameters are bounded by using the proposed controllers. The simulation results demonstrate that the proposed control strategy can drive the chaser to track the position and attitude of the target precisely.

In order to show the advantage of the proposed relative attitude controller 370 (40), the modified Rodrigues parameter-based relative attitude controller is designed by

$$
\boldsymbol{\tau}=-k_{R} \boldsymbol{\sigma}_{e}-k_{w} \boldsymbol{\omega}_{e}+S(\boldsymbol{\omega}) \hat{J} \boldsymbol{\omega}+\hat{J} S(\boldsymbol{\omega}) \boldsymbol{\omega}_{e}-S(\hat{\boldsymbol{\rho}}) \boldsymbol{f}-\hat{\delta}_{A} \tanh \left(\gamma \boldsymbol{e}_{A}\right)
$$

where $\boldsymbol{e}_{A}=\boldsymbol{\omega}_{e}+c_{2} \boldsymbol{\sigma}_{e} ; \boldsymbol{\sigma}_{e}$ is the relative attitude described by modified Rodrigues parameters; the estimations $\hat{J}, \hat{\boldsymbol{\rho}}, \hat{\delta}_{A}$ are still adjusted by adaptive laws (42) and (43); attitude controller parameter is chosen as $k_{R}=60$, while $k_{w}, c_{2}, k_{J}, k_{A}, k_{\rho}, \sigma_{2}, \gamma$ and initial values of estimations are still chosen as controller (40). Moreover, the relative position controller (22) is still employed. By using controller (64), the time history of relative attitude motion is shown in Figure 7. Furthermore, when the feedback gains of controller (64) are enlarged as $k_{R}=k_{w}=160, c_{2}=2$, relative attitude motion simulation result is shown in Figure 8. Comparing Figure 3 with Figures 7 and 8, it is clearly seen that the transient response and steady-state performance of the closed-loop relative attitude system with proposed controller (40) are obviously better than ones with the modified Rodrigues parameter-based relative attitude controller (64).

\section{Conclusion}

385

The six degrees-of-freedom relative motion control problem of a chaser spacecraft approaching a tumbling space target was investigated in this paper. With 
proposing a globally defined relative motion model, robust adaptive controllers were respectively developed for relative position and relative attitude dynamics, in spite of the presence of the kinematic couplings, thrust misalignment, parametric uncertainties and bounded external disturbances. Despite of the independently designing of relative position and attitude controllers, uniformly ultimately boundedness of the six degrees-of-freedom relative motion was derived based on the Lyapunov analysis. A numerical example verified that the chaser can track both the desired relative position and target's attitude precisely by using the proposed controllers. However, the thruster output of the chaser and the approaching time of proximity missions are limited in practice, thus the performance of the closed-loop system may be deteriorated under the control input constraint and finite-time convergence. In future works, an extension of the proposed controller in this study used to handle control input constraint and finite-time convergence problems should be carried out.

\section{References}

[1] Han Y, Biggs, JD, Cui N. Adaptive fault-tolerant control of spacecraft attitude dynamics with actuator failures. J Guid Control Dyn 2015;38(10):2033-42.

[2] Mazinan AH, Pasand M, Soltani B. Full quaternion based finite-time cascade attitude control approach via pulse modulation synthesis for a spacecraft. ISA Trans 2015;58:567-85.

[3] Huo B, Xia Y, Lu K, Fu M. Adaptive fuzzy finite-time fault-tolerant attitude control of rigid spacecraft. J Franklin Inst 2015;352(10):4225-46.

[4] Zhao L, Jia Y. Finite-time attitude tracking control for a rigid spacecraft using time-varying terminal sliding mode techniques. Int J Control 2015;88(6):1150-62.

[5] Gao J, Cai Y. Fixed-time control for spacecraft attitude tracking based on quaternion. Acta Astronaut 2015;115:303-13. 
[6] Zhao L, Jia Y. Neural network-based distributed adaptive attitude synchronization control of spacecraft formation under modified fast terminal sliding mode. Neurocomputing 2016;171:230-41.

[7] Zhou N, Xia Y. Coordination control design for formation reconfiguration of multiple spacecraft. IET Control Theory Appl 2015;9(15):2222-31.

[8] Ghasemi S, Khorasani K. Fault detection and isolation of the attitude control subsystem of spacecraft formation flying using extended Kalman filters. Int J Control 2015;88(10):2154-79.

[9] Mehrabian A, Khorasani K. Distributed and cooperative quaternionbased attitude synchronization and tracking control for a network 425 of heterogeneous spacecraft formation flying mission. J Franklin Inst 2015;352(9):3885-913.

[10] Cabecinhas D, Cunha R, Silvestre C. Almost global stabilization of fullyactuated rigid bodies. Syst Control Lett 2009;58(9):639-45.

[11] Lee T. Exponential stability of an attitude tracking control system on $\mathrm{SO}(3)$ for large-angle rotational maneuvers. Syst Control Lett 2012;61(1):231-7.

[12] Younes AB, Mortari D, Turner JD. Attitude error kinematics. J Guid Control Dyn 2014;37(1):330-U24.

[13] Chaturvedi NA, Sanyal AK, McClamroch NH. Rigid body attitude control. IEEE Control Syst Mag 2011;31(3):30-51.

${ }_{435}$ [14] Bhat SP, Bernstein DS. A topological obstruction to continuous global stabilization of rotational motion and the unwinding phenomenon. Syst Control Lett 2000;39(1):63-70.

[15] Lv Y, Hu Q, Ma G, Zhou J. 6DOF synchronized control for spacecraft formation flying with input constrint and parameter uncertainties. ISA Trans 440 2011;50(4):573-80. 
[16] Bustan D, Pariz N, Hosseini Sani SK. Robust fault-tolerant tracking control design for spacecraft under control input saturation. ISA Trans 2014;53(4):1073-80.

[17] Yuichi I, Takashi K, Tomoyuki N. Nonlinear tracking control of rigid spacecraft under disturbance using PD and PID type $\mathcal{H}_{\infty}$ state feedback. In: Proceedings of the 50th IEEE Conference on Decision and Control \& European Control Conference, IEEE Publication, Piscataway, NJ, 2011, pp. 6184-91.

[18] Xin M, Pan H. Integrated nonlinear optimal control of spacecraft in proximity operations. Int J Control 2010;83(2):347-63.

[19] Hu Q, Li B, Zhang Y. Robust attitude control design for spacecraft under assigned velocity and control constraints. ISA Trans 2013;52(4):480-93.

[20] Zhang F, Duan G. Robust adaptive integrated translation and rotation finite-time control of a rigid spacecraft with actuator misalignment and unknown mass property. Int J Syst Sci 2014;45(5):1007-34.

[21] Zou A. Finite-time output feedback attitude tracking control for rigid spacecraft. IEEE Trans Control Syst Technol 2014;22(1):338-45.

[22] Singla P, Subbarao K, Junkins JL. Adaptive output feedback control for spacecraft rendezvous and docking under measurement uncertainty. J Guid Control Dyn 2006;29(4):892-902.

[23] Subbarao K, Welsh S. Nonlinear control of motion synchronization for satellite proximity operations. J Guid Control Dyn 2008;31(5):1284-94.

[24] Zhang F, Duan G. Integrated translational and rotational finite-time maneuver of a rigid spacecraft with actuator misalignment. IET Control Theory Appl 2012;6(9):1192-204.

[25] Wang X, Yu C. Unit dual quaternion-based feedback linearization tracking problem for attitude and position dynamics. Syst Control Lett 2013;62(3):225-33. 
[26] Wang J, Liang H, Sun Z, Wu S, Zhang S. Relative motion coupled control based on dual quaternion. Aerosp Sci Technol 2013;25(1):102-13.

[27] Wu J, Liu K, Han D. Adaptive sliding mode control for six-DOF relative motion of spacecraft with input constraint. Acta Astronaut 2013;87:64-76.

[28] Pukdeboon C. Inverse optimal sliding mode control of spacecraft with coupled translation and attitude dynamics. Int J Syst Sci 2015;46(13):2421-38.

[29] Mayhew CG, Sanfelice RG, Teel AR. Quaternion-based hybrid control for robust global attitude tracking. IEEE Trans Autom Control 2011;56(11):2555-66.

[30] Lee D, Vukovich G. Adaptive sliding mode control for spacecraft body-fixed hovering in the proximity of an asteroid. Aerosp Sci Technol 2015;46:47183.

[31] Sun L, Huo W. Robust adaptive control of spacecraft proximity maneuvers under dynamic coupling and uncertainty. Adv Space Res 2015;56(10):220617.

[32] Sun L, Huo W. Robust adaptive relative position tracking and attitude synchronization for spacecraft rendezvous. Aerosp Sci Technol 2015;41:2835.

[33] Sun L, Huo W. Nonlinear robust adaptive trajectory tracking control for spacecraft proximity operations. J Aerosp Eng 2015;229(13):2429-40.

[34] Sun L, Huo W. 6-DOF intergrated adaptive backstepping control for spacecraft proximity operations. IEEE Trans Aerosp Electron Syst 2015;51(3):2433-43.

[35] Pan H, Kapila V. Adaptive nonlinear control for spacecraft formation flying with coupled translational and attitude dynamics. In: Proceedings of the 40th IEEE Conference on Decision and Control, IEEE Publication, Piscataway, NJ, 2001, pp. 2057-62. 
[36] Zhang F, Duan G. Integrated relative position and attitude control of spacecraft in proximity operation missions. Int J Automation Computing 2012;9(4):342-51.

[37] Ploycarpou MM. A robust adaptive nonlinear control design. Automatica 1996;32(3):423-27.

[38] Cunha R, Silvestre C, Hespanha J. Output-feedback control for stabilization on SE(3). Syst Control Lett 2008;57(12):1013-22.

[39] Xin M, Pan H. Nonlinear optimal control of spacecraft approaching a tumbling target. Aerosp Sci Technol 2011;15(2):79-89.

[40] Lee T. Robust adaptive attitude tracking on $\mathrm{SO}(3)$ with an application to a quadrotor UAV. IEEE Trans Control Syst Technol 2013;21(5):1924-30.

[41] Sanyal AK, Chaturvedi NA. Almost global robust attitude tracking control of spacecraft in gravity. AIAA Guidiance, Navigation and Control Conference Exhibit, AIAA Paper 2008-6979, 2008.

[42] Koditschek D. Application of a new Lyapunov function to global adaptive tracking. In: Proceedings of the 27th IEEE Conference on Decision and Control, IEEE Publication, Piscataway, NJ, 1988, pp. 63-68.

[43] Kim SG, Crassidis JL, Cheng Y, Fosbury A M, Junkins JL. Kalman filtering for relative spacecraft attitude and position estimation. J Guid Control Dyn 2007;30(1):133-43.

[44] Segal S, Carmi A, Gurfil P. Stereovision-based estimation of relative dynamics between noncooperative satellite: theory and experiments. IEEE Trans Control Syst Technol 2014;22(2):568-84.

[45] Shuster MD. A survey of attitude representations. J Astronautical Sci 520 1993;41(4): 439-517.

[46] Krstic M, Kanellakopoulos I, Kokotovic P. Nonlinear and Adaptive Control Design. New York: John Wiley \& Sons, 1995. 
[47] Adetola V, Guay M. Performance improvement in adaptive control of linearly parameterized nonlinear systems. IEEE Trans Autom Control 2010;55(9):2182-86.

[48] Huang Y, Na J, WU X, Liu X, Guo Y. Adaptive control of nonlinear uncertain active suspension systems with prescribed performance. ISA Trans $2015 ; 54: 145-55$.

[49] Na J, Mahyuddin MN, Herrmann G, Ren X, Barber P. Robust adaptive finite-time parameter estimation and control for robotic systems. Int J Robust Nonlinear Control doi:101002/rnc.3247.

[50] Slotine JJE, Li W. Applied Nonlinear Control. Pretice Hall Englewood Cliffs: NJ, 1991. 


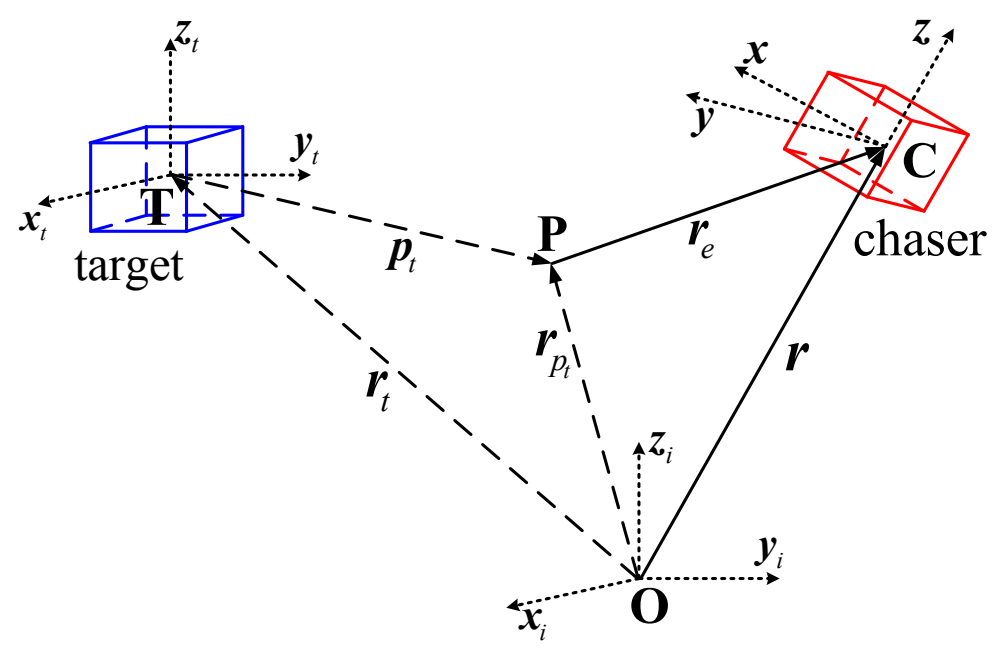

Figure 1: Description of the related frames and vectors.
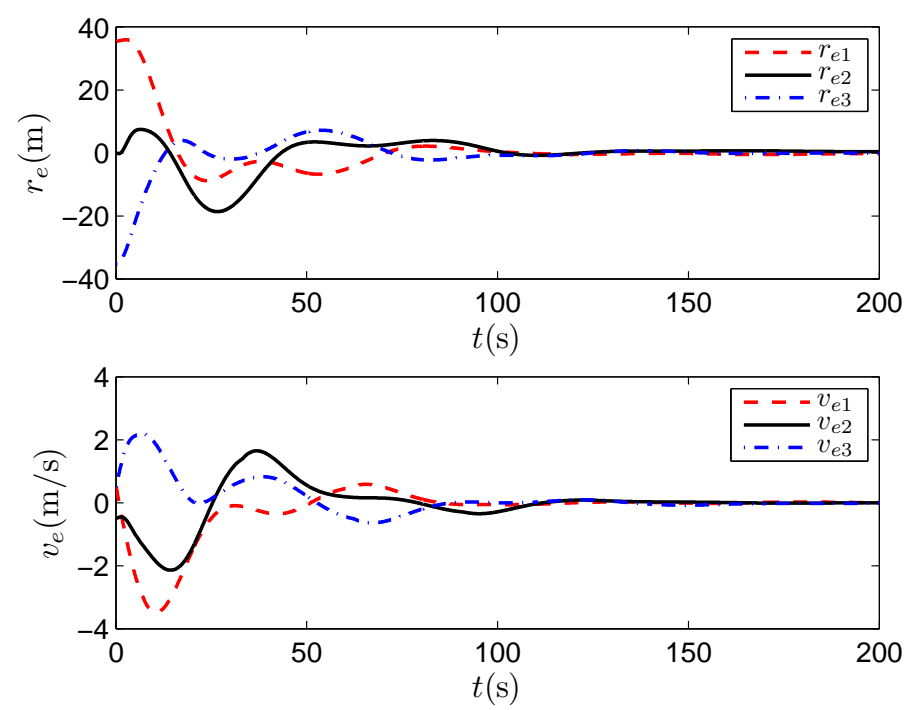

Figure 2: Relative position motion under controller (22). 

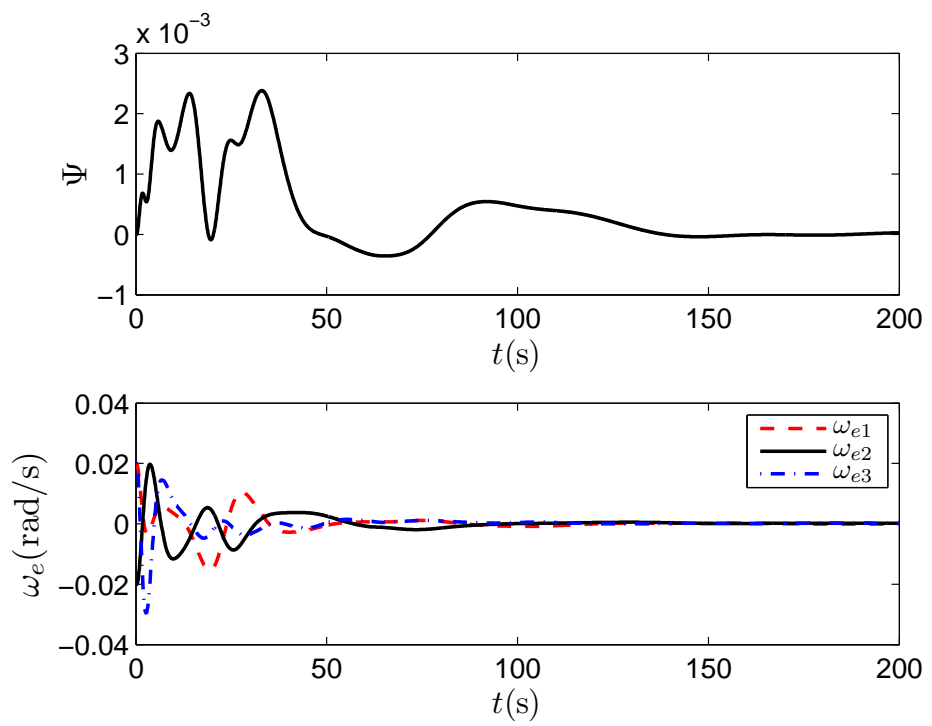

Figure 3: Relative attitude motion under controller (40).
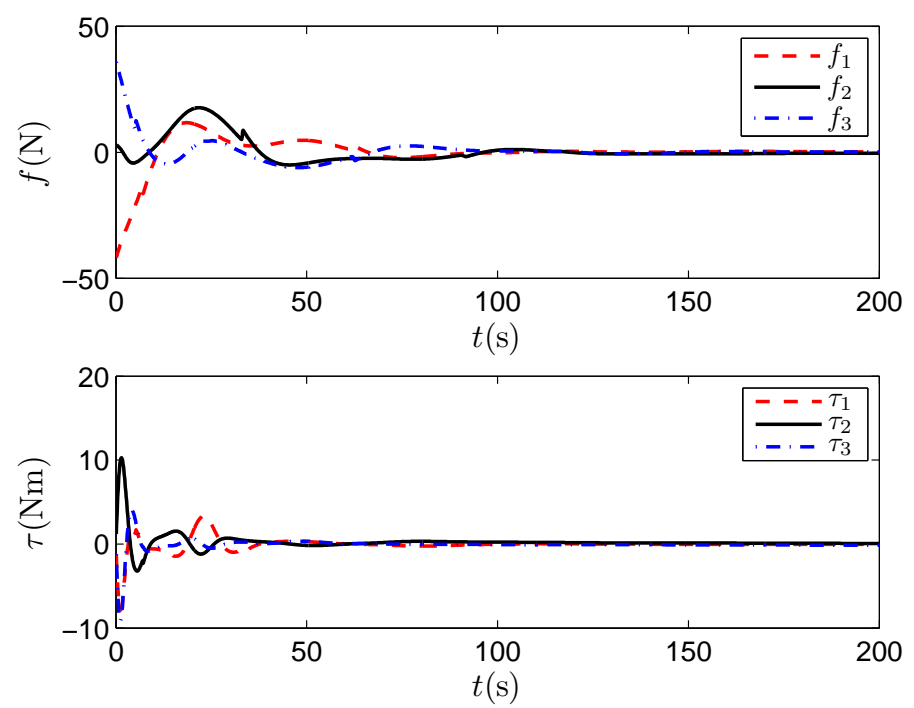

Figure 4: Control forces and torques under controllers (22) and (40). 

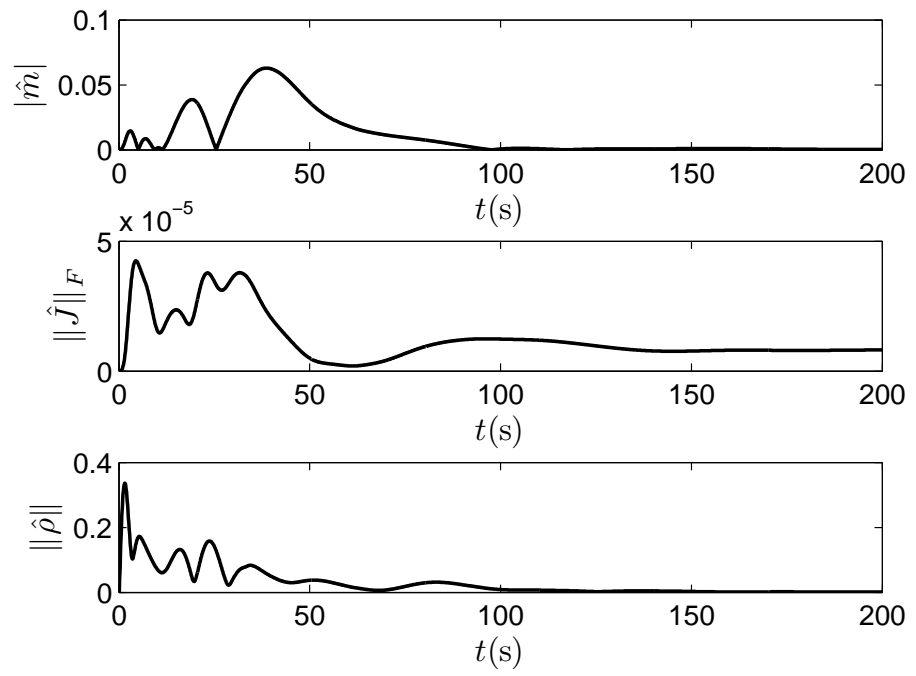

Figure 5: Parameters estimation $\hat{m}, \hat{J}$, and $\hat{\boldsymbol{\rho}}$ under controllers (22) and (40).
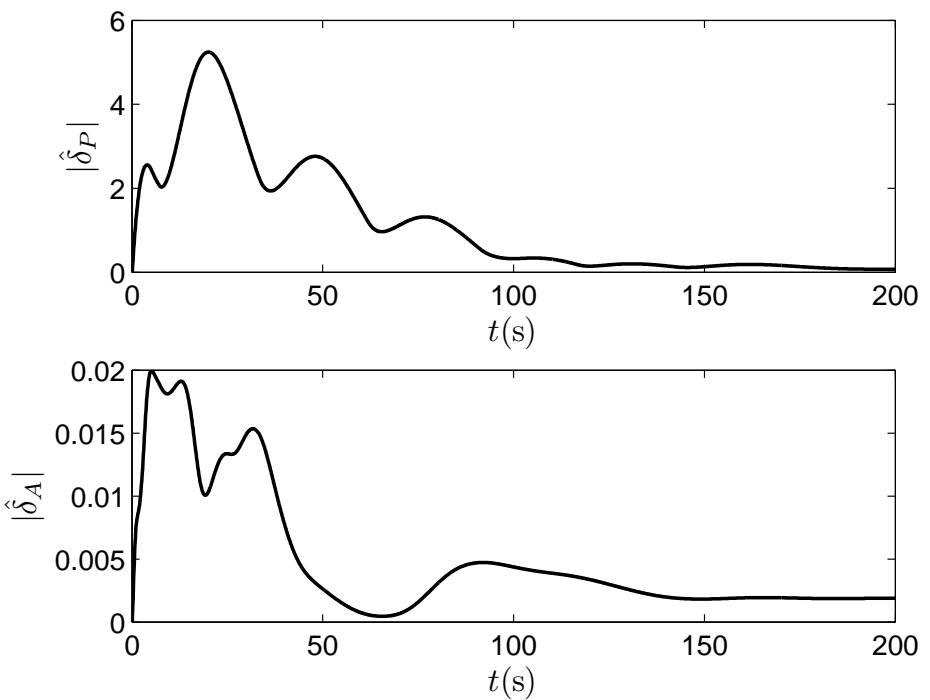

Figure 6: Parameters estimation $\hat{\delta}_{P}$ and $\hat{\delta}_{A}$ under controllers (22) and (40). 

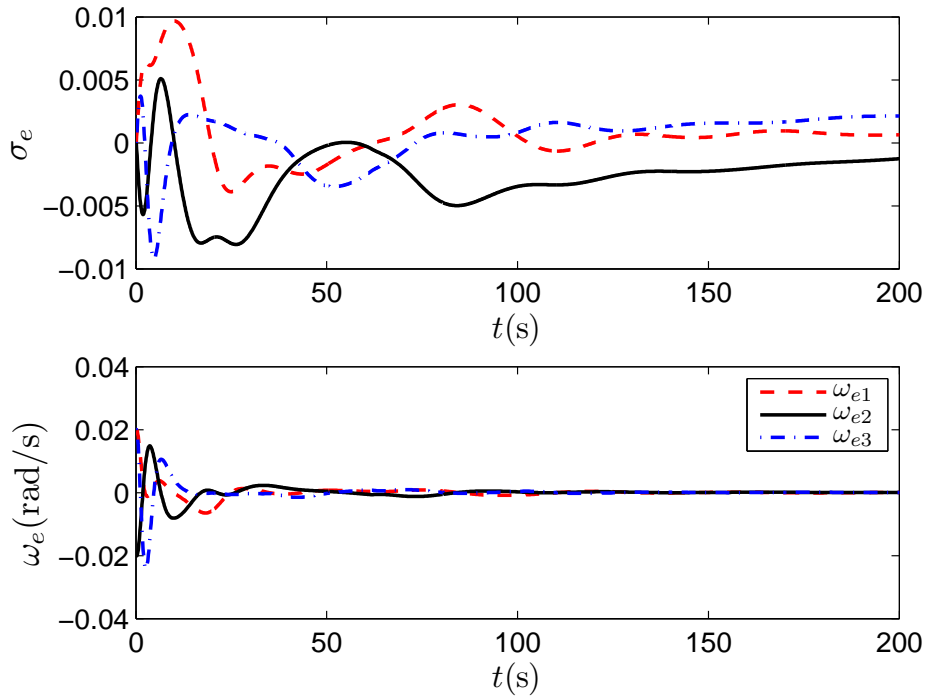

Figure 7: Relative attitude motion under controller (64) with small gains.
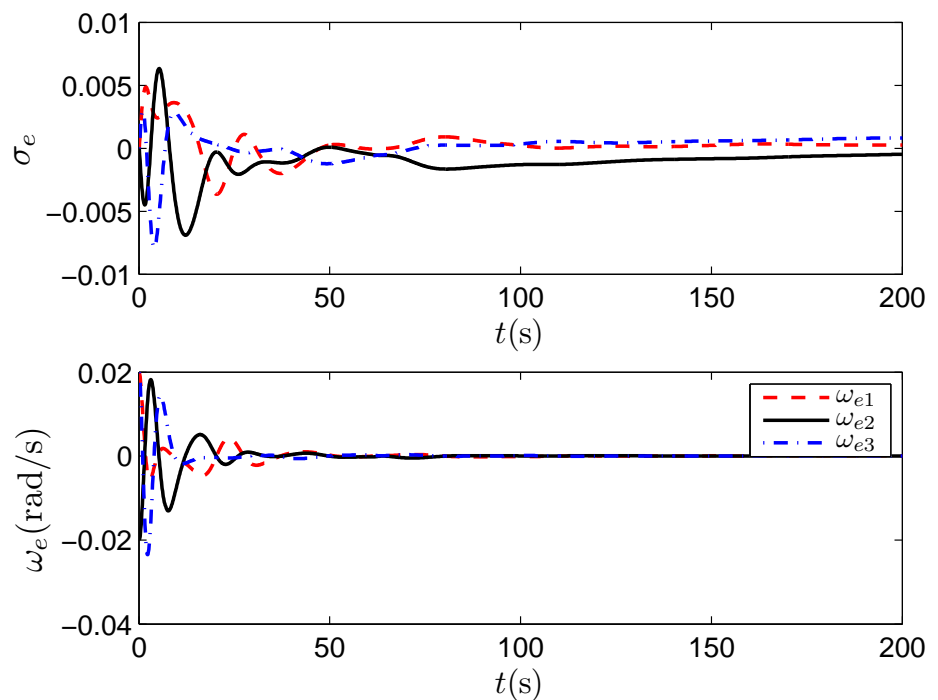

Figure 8: Relative attitude motion under controller (64) with large gains. 